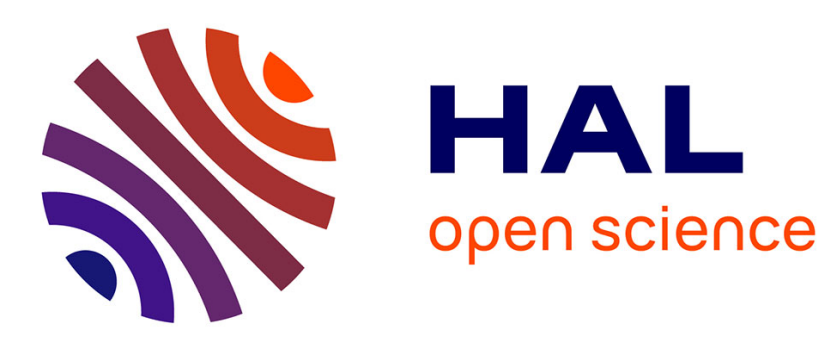

\title{
A simple accurate expression of the potential in electrostatic lenses. Part II : triple cylinder lenses
}

P. Bonjour

\section{To cite this version:}

P. Bonjour. A simple accurate expression of the potential in electrostatic lenses. Part II : triple cylinder lenses. Revue de Physique Appliquée, 1979, 14 (7), pp.715-728. 10.1051/rphysap:01979001407071500 . jpa-00244652

HAL Id: jpa-00244652

https://hal.science/jpa-00244652

Submitted on 1 Jan 1979

HAL is a multi-disciplinary open access archive for the deposit and dissemination of scientific research documents, whether they are published or not. The documents may come from teaching and research institutions in France or abroad, or from public or private research centers.
L'archive ouverte pluridisciplinaire HAL, est destinée au dépôt et à la diffusion de documents scientifiques de niveau recherche, publiés ou non, émanant des établissements d'enseignement et de recherche français ou étrangers, des laboratoires publics ou privés. 


\title{
A simple accurate expression of the potential in electrostatic lenses. Part II : triple cylinder lenses
}

\author{
P. Bonjour \\ Institut d'Electronique Fondamentale (*), Bâtiment 220, Université Paris-Sud, 91405 Orsay Cedex, France
}

(Reçu le 16 février 1979, révisé le 3 avril 1979, accepté le 6 avril 1979)

Résumé. - On présente une méthode simple et précise de calcul du potentiel dans les lentilles formées de trois cylindres séparés par deux intervalles finis sous la forme d'un développement en série de fonctions de Bessel.

Les éléments optiques de lentilles mécaniquement symétriques et unipotentielles ou électriquement asymétriques ont été calculés. Les valeurs obtenues sont en très bon accord avec celles obtenues par Read au moyen de la méthode de distribution de charges.

L'influence de l'asymétrie mécanique sur les propriétés optiques a été également étudiée, et un résultat important mis en évidence : les propriétés optiques dépendent essentiellement de la distance entre les plans médians des deux gaps et très peu de la longueur de ces gaps.

\begin{abstract}
A simple accurate method is presented of calculating the potential in lenses composed of three cylinders separated by two finite gaps under the form of an expansion of Bessel functions.

The optical elements of mechanically symmetrical lenses, and, either unipotential, or electrically asymmetrical ones, have been computed. The results agree very well with those obtained by Read by means of the charge distribution method.

The influence of the mechanical asymmetry on the optical properties has also been studied, and an important property has been pointed out : the optical properties depend essentially on the distance between the median planes of the two gaps and very little on the lengths of these gaps.
\end{abstract}

1. Introduction. - Three-cylinder lenses are widely used in electron microscopy, in focussing beams in cathode ray tubes and generally in handling beams of charged particles. They are easy to build and adjust, and their insensitivity to the $e / m$ ratio of the focussed particles make them very convenient. In these lenses, the potentials of the three cylinders can be different, but most of them are of the unipotential type, in which the potentials of the outer cylinders are identical. The potential of the central cylinder may then be either greater or smaller than that of the two others, and, from the same mechanical disposition of the electrodes, very varied focussing properties may result.

The design of these lenses has been carried out mainly by computing the axial potential by one of the three following methods :

- Laplace's equation has been solved by the five or nine point relaxation method, in which the conver-

(*) Laboratoire associé au C.N.R.S. gence is accelerated by the overrelaxation factor, by many authors, for instance Saito et al. [1, 2], who have calculated lenses in which the outer cylinders are held to equal or different potentials.

- Adams and Read [3, 4] have applied the charge distribution method. The potential of a three-cylinder lens can be obtained, either by applying the method directly to the three cylinders, or by assuming that a three-cylinder lens is a superposition of two doublecylinder ones shifted along the common axis of symmetry.

- Laplace's equation can also be solved by means of Fourier-Bessel series, but, as an electrostatic lens is an open system, the difficulty is to write realistic boundary conditions. When the potential is known along a particular curve, for example between the cylinders along their common edge, the coefficients of the series can be computed for a unipotential lens by Fourier analysis [5], but the method in itself is insufficient to fix the potential between the cylinders which depends on an additional condition. 
In a recent paper ([6], referred to as Part I thereinafter), we have presented an accurate method of solving Laplace's equation within a lens whose two cylinders are separated by a finite distance. Assuming that the potential along the outer edge of the cylinders in the gap follows a third degree law, we use a variational principle to obtain the potential as a series of Bessel functions. Then the axial potential is calculated and used to determine the optical properties of the lens. We will here apply this method to three-cylinder lenses.

2. Calculation of the potential. - We consider the three-cylinder system shown in figure 1 . The three cylinders are held at potentials $V 1, V 2$ and $V 3$, and the origin of the potentials corresponds to a zero electron velocity. To make things easier later on, it is advisable to set $V_{g}=V 2-V 1$ and $V_{d}=V 3-V 2$.

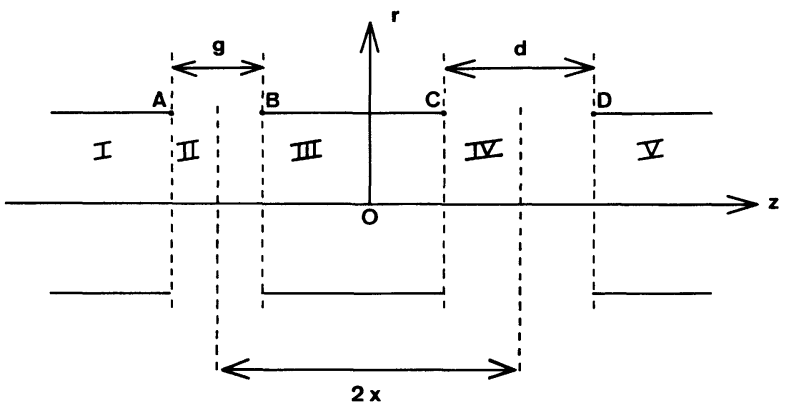

Fig. 1. - The geometry of a triple cylinder lens, showing the system of coordinates and the dimensions. The origin $O$ is placed half-way between the two gaps median plane, and the plane which is perpendicular in $O$ to the axis is used as a reference plane for the position of the cardinal elements.

The cylinders are separated by gaps whose lengths are respectively $G$ for the left-hand gap and $D$ for the right-hand one. The distance between the median planes of the gaps is $2 X$. We use cylindrical coordinates with their origin placed half-way between the median planes of the gaps, and the common radius $R$ of the cylinders is chosen as the unit of length. The normalized lengths are written with lower-case letters $(g=G / R, x=X / R, \ldots)$.

Between the points A and B of the left-hand gap, the potential is represented by means of a third degree polynomial

$$
\begin{aligned}
V(z)= & V 1+V_{g}\left[\frac{a+1}{g}(z+x+g / 2)\right. \\
& \left.-\frac{3 a}{g^{2}}(z+x+g / 2)^{2}+\frac{2 a}{g^{3}}(z+x+g / 2)^{3}\right] .
\end{aligned}
$$

This potential is equal to $V 1$ for $z=-x-g / 2$ and to $V 2$ for $z=-x+g / 2 ; a$ is a parameter which must be determined.

Between the points $\mathrm{C}$ and $\mathrm{D}$ of the right-hand gap, the potential is represented by means of another third degree polynomial

$$
\begin{aligned}
V(z)= & V 2+V_{d}\left[\frac{b+1}{d}(z-x+d / 2)\right. \\
& \left.-\frac{3 b}{d^{2}}(z-x+d / 2)^{2}+\frac{2 b}{d^{2}}(z-x+d / 2)^{3}\right] .
\end{aligned}
$$

This potential is equal to $V 2$ for $z=x-d / 2$ and to $V 3$ for $z=x+d / 2 ; b$ is a parameter to be determined.

As we have shown in Part I, potentials (1) and (2) can be expressed by means of the functions $V_{n}(z)$

$$
\begin{array}{ll}
V_{n}(z)=0 & z \leqslant 0 \\
V_{n}(z)=z^{n} & z \geqslant 0
\end{array}
$$

and (1) becomes

$$
\begin{aligned}
V(z)= & V 1+V_{g}\left\{\frac{a+1}{g}\left[V_{1}(z+x+g / 2)-V_{1}(z+x-g / 2)\right]\right. \\
& \left.-\frac{3 a}{g^{2}}\left[V_{2}(z+x+g / 2)+V_{2}(z+x-g / 2)\right]+\frac{2 a}{g^{3}}\left[V_{3}(z+x+g / 2)-V_{3}(z+x-g / 2)\right]\right\} .
\end{aligned}
$$

Expression (3) represents a potential equal to $V 1$ for $z \leqslant-x-g / 2$ and to $V 2$ for $z \geqslant-x+g / 2$, and varying according to (1) for $-x-g / 2 \leqslant z \leqslant-x+g / 2$. In the same way (2) becomes

$$
\begin{aligned}
V(z)= & V 2+V_{d}\left\{\frac{b+1}{d}\left[V_{1}(z-x+d / 2)-V_{1}(z-x-d / 2)\right]\right. \\
& \left.-\frac{3 b}{d^{2}}\left[V_{2}(z-x+d / 2)+V_{2}(z-x-d / 2)\right]+\frac{2 b}{d^{3}}\left[V_{3}(z-x+d / 2)-V_{3}(z-x-d / 2)\right]\right\} .
\end{aligned}
$$


Expression (4) represents a potential equal to $V 2$ for $z \leqslant x-d / 2$ and to $V 3$ for $z \geqslant x+d / 2$, and varying according to (2) for $x-d / 2 \leqslant z \leqslant x+d / 2$. Superposing the two potentials represented by (3) and (4), we obtain

$$
\begin{aligned}
V(z)=V 1 & +V_{g}\left\{\frac{a+1}{g}\left[V_{1}(z+x+g / 2)-V_{1}(z+x-g / 2)\right]\right. \\
& \left.-\frac{3 a}{g^{2}}\left[V_{2}(z+x+g / 2)+V_{2}(z+x-g / 2)\right]+\frac{2 a}{g^{3}}\left[V_{3}(z+x+g / 2)-V_{3}(z+x-g / 2)\right]\right\} \\
& +V_{d}\left\{\frac{b+1}{d}\left[V_{1}(z-x+d / 2)-V_{1}(z-x-d / 2)\right]-\frac{3 b}{d^{2}}\left[V_{2}(z-x+d / 2)+V_{2}(z-x-d / 2)\right]\right. \\
& \left.+\frac{2 b}{d^{3}}\left[V_{3}(z-x+d / 2)-V_{3}(z-x-d / 2)\right]\right\}
\end{aligned}
$$

Expression (5) represents the potential along the outer edges of the cylinders $(r=1)$ and is equal to $V 1$ for $z \leqslant-x-g / 2$, varies according to (1) for $-x-g / 2 \leqslant z \leqslant-x+g / 2$, is equal to $V 2$ for

$$
-x+g / 2 \leqslant z \leqslant x-d / 2,
$$

varies according to (2) for $x-d / 2 \leqslant z \leqslant x+d / 2$ and is equal to $V 3$ for $z \geqslant x+d / 2$.

The solution of Laplace's equation within the three-cylinder system, when the potential for $r=1$ is given by (5), is obtained by using the $V_{n}(r, z)$ functions of Bertram [7], as we have shown in Part I, and it is expressed as

$$
\begin{aligned}
V(r, z)=V 1+V_{g}\left\{\frac { a + 1 } { g } \left[V_{1}(r, z+x+g / 2)\right.\right. & \left.-V_{1}(r, z+x-g / 2)\right] \\
& -\frac{3 a}{g^{2}}\left[V_{2}(r, z+x+g / 2)+V_{2}(r, z+x-g / 2)\right] \\
& \left.+\frac{2 a}{g^{3}}[V(r, z+x+g / 2)-V(r, z+x-g / 2)]\right\} \\
& +V_{d}\left\{\frac{b+1}{d}\left[V_{1}(r, z-x+d / 2)-V_{1}(r, z-x-d / 2)\right]\right. \\
& -\frac{3 b}{d^{2}}\left[V_{2}(r, z-x+d / 2)+V_{2}(r, z-x-d / 2)\right] \\
& \left.+\frac{2 a}{d^{3}}\left[V_{3}(r, z-x+d / 2)-V_{3}(r, z-x-d / 2)\right]\right\} .
\end{aligned}
$$

3. Calculation of the parameters $\boldsymbol{a}$ and $\boldsymbol{b}$. - When the potential within the lens is expressed by (6), the electrostatic energy stored inside it is written as

$$
\begin{aligned}
E=\left(\frac{a+1}{g}\right)^{2} W_{11}+\left(\frac{3 a}{g^{2}}\right)^{2} & W_{22}+\left(\frac{2 a}{g^{3}}\right)^{2} W_{33}-\frac{6 a(a+1)}{g^{3}} W_{12} \\
& +\frac{4 a(a+1)}{g^{4}} W_{13}-\frac{12 a^{2}}{g^{5}} W_{23}+\left(\frac{b+1}{d}\right)^{2} X_{11}+\left(\frac{2 b}{d^{2}}\right)^{2} X_{22} \\
& +\left(\frac{2 b}{d^{3}}\right)^{2} X_{33}-\frac{6 b(b+1)}{d^{3}} X_{12}+\frac{4 b(b+1)}{d^{4}} X_{13}-\frac{12 b^{2}}{d^{5}} X_{23} \\
& +\frac{2(a+1)(b+1)}{g d} Y_{11}-\frac{6 b(a+1)}{g d^{2}} Y_{12}-\frac{6 a(b+1)}{g^{2} d} Y_{21}+\frac{4 b(a+1)}{g d^{3}} Y_{13} \\
& +\frac{4 a(b+1)}{g^{3} d} Y_{31}+\frac{18 a b}{g^{2} d^{2}} Y_{22}+\frac{8 a b}{g^{3}} Y_{33}-\frac{12 a b}{g^{2} d^{3}} Y_{23}-\frac{12 a b}{g^{3} d^{2}} Y_{32} .
\end{aligned}
$$

The terms $W_{i j}$ come from the functions $V_{n}(r, z-x \pm g / 2)$ representing the potential created by the lefthand gap. The terms $X_{i j}$ come from the functions $V_{n}(r, z-x \pm d / 2)$ related to the right-hand gap. The terms $Y_{i j}$ come from the product of terms of the form $V_{n}(r, z+x \pm g / 2) V_{n}(r, z-x \pm d / 2)$, and they represent the energetic interaction between the two gaps. 
The total energy $\mathcal{E}$ is a quadratic form in the parameters $a$ and $b$; it can be written as the general expression

$$
\varepsilon=A a^{2}+2 B a b+C b^{2}+2 D a+2 E b+F
$$

$A, B, \ldots F$ being functions of the terms $W_{i j}, X_{i j}$ and $Y_{i j}$. The values of the parameters $a$ and $b$ are obtained from the condition that $\delta$ is an extremum with respect to variations of $a$ and $b$. By writing

$$
\frac{\partial \mathcal{E}}{\partial a}=0 \text { and } \frac{\partial \mathcal{E}}{\partial b}=0
$$

the following values of $a$ and $b$ are obtained

$$
\begin{aligned}
& a=\frac{B E-C D}{A C-B^{2}} \\
& b=\frac{B D-A E}{A C-B^{2}} .
\end{aligned}
$$

The total energy $\varepsilon$ is obtained by integrating the square of the electric field modulus over the system inner volume, and this gives the following expression

$$
\begin{aligned}
E=\int_{0}^{1} \int_{-\infty}^{-x-g / 2}\left[\left(\frac{\partial V_{\mathrm{I}}}{\partial z}\right)^{2}\right. & \left.+\left(\frac{\partial V_{\mathrm{I}}}{\partial r}\right)^{2}\right] \mathrm{d} r \mathrm{~d} z+ \\
& +\int_{0}^{1} \int_{-x-g / 2}^{-x+g / 2}\left[\left(\frac{\partial V_{\mathrm{II}}}{\mathrm{d} z}\right)^{2}+\left(\frac{\partial V_{\mathrm{II}}}{\partial r}\right)^{2}\right] \mathrm{d} r \mathrm{~d} z+\int_{0}^{1} \int_{-x+g / 2}^{x-d / 2}\left[\left(\frac{\partial V_{\mathrm{III}}}{\partial z}\right)^{2}+\left(\frac{\partial V_{\mathrm{III}}}{\partial r}\right)^{2}\right] \mathrm{d} r \mathrm{~d} z \\
& +\int_{0}^{1} \int_{x-d / 2}^{x+d / 2}\left[\left(\frac{\partial V_{\mathrm{IV}}}{\partial z}\right)^{2}+\left(\frac{\partial V_{\mathrm{IV}}}{\partial r}\right)^{2}\right] \mathrm{d} r \mathrm{~d} z+\int_{0}^{1} \int_{x+d / 2}^{\infty}\left[\left(\frac{\partial V_{\mathrm{V}}}{\partial z}\right)^{2}+\left(\frac{\partial V_{\mathrm{V}}}{\partial r}\right)^{2}\right] \mathrm{d} r \mathrm{~d} z
\end{aligned}
$$

$V_{\mathrm{I}}, V_{\mathrm{II}}, \ldots, V_{\mathrm{V}}$ express the potential in the five regions of figure 1, obtained by substituting the appropriate expression for Bertram' functions $V_{n}(r, z)$ in (6).

After some calculations, the following expressions for the $W_{i j}$ are obtained

$$
\begin{aligned}
& W_{11} / V_{g}^{2}=\frac{g}{2}-2 \sum \frac{1}{\mu^{3}}[1-\exp (-\mu g)] \\
& W_{22} / V_{g}^{2}=\frac{2 g^{3}}{3}+8 g \sum \frac{1}{\mu^{4}}[2-\exp (-\mu g)]+8 \sum \frac{1}{\mu^{5}}[1+\exp (-\mu g)] \\
& W_{33} / V_{g}^{2}=\frac{9 g^{5}}{10}+48 g^{3} \sum \frac{1}{\mu^{4}}-36 g^{2} \sum \frac{1}{\mu^{5}}[1-\exp (-\mu g)]+72 g \sum \frac{1}{\mu^{6}}-72 \sum \frac{1}{\mu^{7}}[1-\exp (-\mu g)] \\
& W_{12} / V_{g}^{2}=\frac{g^{2}}{2}-2 g \sum \frac{1}{\mu^{3}}[1-\exp (-\mu g)]+4 \sum \frac{1}{\mu^{4}}[1-\exp (-\mu g)] \\
& W_{13} / V_{g}^{2}=\frac{g^{3}}{2}-3 g^{2} \sum \frac{1}{\mu^{3}}[1-\exp (-\mu g)]+12 g \sum \frac{1}{\mu^{4}}-12 \sum \frac{1}{\mu^{5}}[1-\exp (-\mu g)] \\
& W_{23} / V_{g}^{2}=\frac{3 g^{4}}{4}+24 g^{2} \sum \frac{1}{\mu^{4}}+6 g^{2} \sum \frac{1}{\mu^{4}}[1-\exp (-\mu g)]-12 g \sum \frac{1}{\mu^{5}}[1-\exp (-\mu g)]
\end{aligned}
$$

The summations have to be taken over all the positive roots $\mu$ of the equation $J_{0}(x)=0$.

It can be noticed that these expressions are the same as the expressions (8) in Part I and, therefore, the $W_{i j}$ terms exactly express the contribution of the left-hand gap alone in the expression of the energy. The $X_{i j}$ terms are obtained from the $W_{i j}$ terms of the same indices by substituting $V_{d}$ for $V_{g}$ and $d$ for $g$ in the expressions (9). 
For the $Y_{i j}$ terms, the expressions are as follows

$Y_{11} / V_{g} V_{d}=\sum \exp (-2 \mu x)\left\{2 \sinh \mu g / 2 \sinh \mu d / 2 \frac{\exp (-\mu g)+\exp (-\mu d)+4}{\mu^{3}}\right.$

$$
\left.-\frac{2}{\mu^{3}} \exp (-\mu g / 2) \sinh \mu d / 2 \sinh \mu g-\frac{2}{\mu^{3}} \exp (-\mu d / 2) \sinh \mu g / 2 \sinh \mu d\right\}
$$

$Y_{22} / V_{g} V_{d}=\sum \exp (-2 \mu x)\left\{8 \cosh \mu g / 2 \cosh \mu d / 2 \frac{\exp (-\mu g)+\exp (-\mu d)}{\mu^{5}}\right.$

$$
\left.\begin{array}{l}
+\frac{8 g}{\mu^{4}} \exp (\mu g / 2) \cosh \mu d / 2-\frac{8 d}{\mu^{4}} \exp (-\mu d / 2) \cosh \mu g / 2 \\
+\frac{8}{\mu^{5}} \exp (-\mu g / 2) \cosh \mu d / 2 \sinh \mu g+\frac{8}{\mu^{5}} \exp (-\mu d / 2) \cosh \mu g / 2 \sinh \mu d
\end{array}\right\}
$$

$Y_{33} / V_{g} V_{d}=\sum \exp (-2 \mu x)\left\{72 \sinh \mu g / 2 \sinh \mu d / 2 \frac{\exp (-\mu g)+\exp (-\mu d)+4}{\mu^{7}}\right.$

$$
\left.\begin{array}{l}
-\frac{72}{\mu^{7}} \exp (-\mu g / 2) \sinh \mu d / 2 \sinh \mu g-\frac{36 d^{2}}{\mu^{5}} \exp (-\mu d / 2) \sinh \mu g / 2 \\
-\frac{72}{\mu^{7}} \exp (-\mu d / 2) \sinh \mu g / 2 \sinh \mu d+\frac{36 g^{2}}{\mu^{5}} \exp (\mu g / 2) \sinh \mu d / 2
\end{array}\right\}
$$

$Y_{12} / V_{g} V_{d}=\sum \exp (-2 \mu x)\left\{4 \sinh \mu g / 2 \cosh \mu d / 2 \frac{\exp (-\mu g)+\exp (-\mu d)+2}{\mu^{4}}\right.$

$$
\begin{aligned}
& -\frac{4}{\mu^{4}} \exp (-\mu g / 2) \cosh \mu d / 2 \sinh \mu g-\frac{4 d}{\mu^{3}} \exp (-\mu d / 2) \sinh \mu g / 2 \\
& \left.+\frac{4}{\mu^{4}} \exp (-\mu d / 2) \sinh \mu g / 2 \sinh \mu d\right\}
\end{aligned}
$$

$Y_{21} / V_{g} V_{d}=\sum \exp (-2 \mu x)\left\{4 \cosh \mu g / 2 \sinh \mu d / 2 \frac{\exp (-\mu g)+\exp (-\mu d)+2}{\mu^{4}}\right.$

$$
\begin{aligned}
& -\frac{4}{\mu^{4}} \exp (-\mu d / 2) \cosh \mu g / 2 \sinh \mu d+\frac{4 g}{\mu^{3}} \exp \mu g / 2 \sinh \mu d / 2 \\
& \left.+\frac{4}{\mu^{4}} \exp (-\mu g / 2) \sinh \mu d / 2 \sinh \mu g\right\}
\end{aligned}
$$

$$
\begin{aligned}
& Y_{13} / V_{g} V_{d}=\sum \exp (-2 \mu x)\left\{12 \sinh \mu g / 2 \sinh \mu d / 2 \frac{\exp (-\mu g)+\exp (-\mu d)+4}{\mu^{5}}\right. \\
& -\frac{6 d^{2}}{\mu^{3}} \exp (-\mu d / 2) \sinh \mu g / 2-\frac{12}{\mu^{5}} \exp (-\mu g / 2) \sinh \mu d / 2 \sinh \mu g \\
& \left.-\frac{12}{\mu^{5}} \exp (-\mu d / 2) \sinh \mu g / 2 \sinh \mu d\right\}
\end{aligned}
$$

$$
\begin{aligned}
Y_{31} / V_{g} V_{d}=\sum \exp (-2 \mu x)\left\{12 \sinh \mu g / 2 \sinh \mu d / 2 \frac{\exp (-\mu g)+\exp (-\mu d)+4}{\mu^{5}}\right. & \\
& +\frac{6 g^{2}}{\mu^{3}} \exp (\mu g / 2) \sinh \mu d / 2-\frac{12}{\mu^{5}} \exp (-\mu g / 2) \sinh \mu d / 2 \sinh \mu g \\
& \left.-\frac{12}{\mu^{5}} \exp (-\mu d / 2) \sinh \mu g / 2 \sinh \mu d\right\}
\end{aligned}
$$




$$
\begin{gathered}
Y_{23} / V_{g} V_{d}=\sum \exp (-2 \mu x)\left\{\begin{array}{c}
24 \cosh \mu g / 2 \sinh \mu d / 2 \frac{\exp (-\mu g)+\exp (-\mu d)+2}{\mu^{6}} \\
-\frac{12 d^{2}}{\mu^{4}} \exp (-\mu d / 2) \cosh \mu g / 2+\frac{24 g}{\mu^{5}} \exp (\mu g / 2) \sinh \mu d / 2
\end{array}\right. \\
\left.\quad-\frac{24}{\mu^{6}} \exp (-\mu g / 2) \sinh \mu d / 2 \sinh \mu g-\frac{24}{\mu^{6}} \exp (-\mu d / 2) \cosh \mu g / 2 \sinh \mu d\right\} \\
Y_{32} / V_{g} V_{d}=\sum \exp (-2 \mu x)\left\{\begin{array}{c}
24 \sinh \mu g / 2 \cosh \mu d / 2 \frac{\exp (-\mu g)+\exp (-\mu d)+2}{\mu^{6}} \\
+\frac{12 g^{2}}{\mu^{4}} \exp (\mu g / 2) \cosh \mu d / 2-\frac{24 d}{\mu^{5}} \exp (-\mu d / 2) \sinh \mu g / 2 \\
\left.+\frac{24}{\mu^{6}} \exp (-\mu d / 2) \sinh \mu g / 2 \sinh \mu d-\frac{24}{\mu^{6}} \exp (-\mu g / 2) \cosh \mu d / 2 \sinh \mu g\right\} .
\end{array}\right.
\end{gathered}
$$

It must be noticed that the $Y_{i j}$ terms tend to zero when the distance between the two gaps increases, and for a very great value of $x$, the energy $\delta$ becomes the arithmetical sum of the energies located in the two gaps. This justifies the approximation which considers a three-cylinder lens as the superposition of two properly shifted double-cylinder lenses when the gaps are not very close.

The calculation of the parameters $a$ and $b$ by means of (9) and (10), then (8) and that of the potential from (6), is achieved by using the methods explained in Part I.

4. Properties of the parameters $a$ and $b$. - The values of the parameters $a$ and $b$ depend in a nonobvious way upon the values of the quantities $V_{g}, V_{d}$, $g, d$ and $x$. They do not depend on the absolute values of $V 1, V 2$ and $V 3$, but only on their ratios. A numerical calculation has revealed the following properties of $a$ and $b$. To make things easier later on, we will call a lens in which $V_{g}=V_{d}$, electrically symmetrical, and a lens in which $g=d$, mechanically symmetrical, thus the definition of an electrically or mechanically asymmetrical lens follows.

1) In an electrically and mechanically symmetrical lens, the parameters $a$ and $b$ are equal and their values are independent of the ratio $V 2 / V 1$.

2) In a lens which is electrically symmetrical and mechanically asymmetrical, the parameters $a$ and $b$ are different, but their values remain independent of the ratio $V 2 / V 1$.

3) In an electrically asymmetrical lens, whatever its mechanical arrangement, the parameters $a$ and $b$ are different, and their values vary with the ratios $V 2 / V 1$ and $V 3 / V 1$.

The calculation of the parameter $a(=b)$ for an electrically and mechanically symmetrical lens gives the values shown in figure 2 as a function of the $x / g$ ratio. The three curves which have been plotted

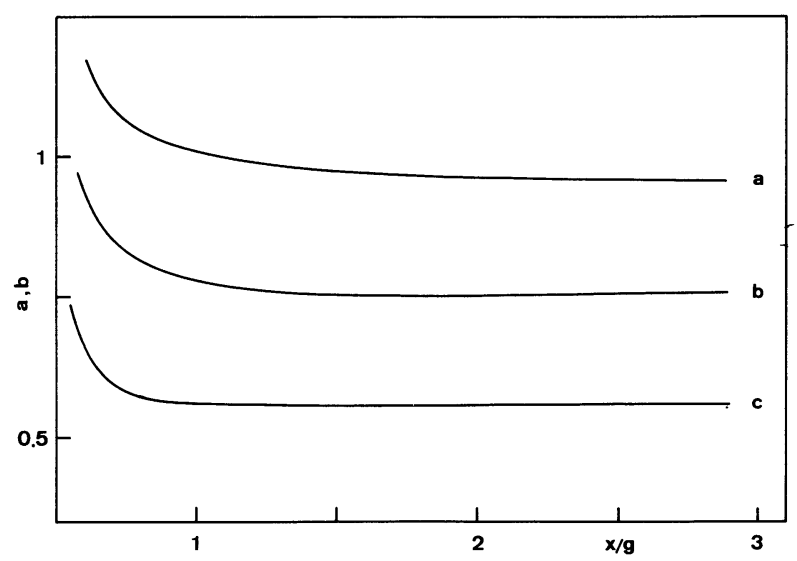

Fig. 2. - The values of the parameters $a=b$ for a mechanically and electrically symmetrical lens as a function of the $x / g$ ratio. The curves correspond to $g=0.2$ (curve $a$ ), $g=1(b)$ and $g=2(c)$.

correspond respectively to the three values $g=0.2$, 1 and 2. The values of $a$ and $b$ are infinite for $x / g=0.5$ and decrease to a finite limit when $x / g$ tends to infinity. A comparison with figure 2 in Part I shows that this limiting value is precisely the same as the one calculated for a single gap.

From these curves, it is equally possible to estimate the error made when, in order to represent the potential of a three-cylinder lens, the potentials of two properly shifted double cylinder lenses are superposed. For instance, figure 2 shows that, for the lens $g=1$, $a$ differs by more than $10 \%$ from its limiting value when $x / g$ is less than 0.72 . The values of the axial potential are modified through expression (6); for instance, in the gap centres, the slope of the potential curve, which is proportional to $a+1$, is increased of about $4 \%$. In a general way, the potential varies more rapidly over the gaps than it does in the case of a double-cylinder lens gap, and for the same value of the axial potential, the curve slope is greater.

By using an approximate formula to evaluate the 
focal length, such as the expression $4.15 \mathrm{c}$ in Grivet's book [8]

$$
\frac{1}{f}=\frac{3}{16} \int\left(\frac{V^{\prime}}{V}\right)^{2} \mathrm{~d} z
$$

it can be noticed that the focal length will be modified by about $8 \%$. Of course, this way of reasoning is not very accurate, but it suggests a lower limit for the gap width, beneath which erroneous results will be obtained when a three-cylinder lens is replaced by two shifted double-cylinder ones.

5. Optical properties of electrically symmetrical lenses. - The cardinal elements and the spherical aberration coefficient are calculated from the axial potential as shown in Part I, for electrons crossing from the region of $V 1$ potential to the region of $V 3$ potential. We have calculated the focal lengths, object $F 1$ and image $F 2$, the positions of the object focus $Z F 1$ and of the image focus $Z F 2, S P 1$ is the spherical aberration constant for an image located at infinity, and $S P 2$ is the limit of the product $C_{\mathrm{s}} M^{4}$ (spherirical aberration constant $C_{\mathrm{s}}$ and magnification $M$ ) when the object moves to infinity on the left.

Table I has been computed for an electrically and mechanically symmetrical lens (unipotential lens) in which $g=0.2$. In this type of lens, $F 1=F 2$, $Z F 1=Z F 2, S P 1=S P 2$ and these quantities only depend on the ratio $V 2 / V 1$. The computation has been made for three $x$ values : $0.2,0.5$ and 1 . The values of $V 2 / V 1$ vary by steps of 0.1 between -1 and 1 and according to a geometrical progression with a ratio $(10)^{1 / 12}$ outside this range. When $V 2$ decreases and becomes less than $V 1$, there is a value of

Table I. - Values of the cardinal elements and of the spherical aberration constant as a function of the voltage ratio $V 2 / V 1$ for a unipotential lens $g=d=0.2$. The first three columns correspond to $x=0.2$, the following three to $x=0.5$, and the last three to $x=1$. The number $x . x x x \pm y y$ should be read $x \cdot x x x 10^{ \pm y y}$.

\begin{tabular}{|c|c|c|c|c|c|c|c|c|c|}
\hline$V 2 / V 1$ & $F 1$ & $Z F 1$ & $S P 1$ & $F 1$ & $Z F 1$ & $S P 1$ & $F 1$ & $Z F 1$ & $S P 1$ \\
\hline- & - & - & - & - & - & - & - & - & - \\
\hline-2.7 & $6.42-01$ & $2.58-01$ & $5.02+00$ & & & & & & \\
\hline-2.2 & $1.34+00$ & $1.21+00$ & $1.71+01$ & & & & & & \\
\hline-1.8 & $2.34+00$ & $2.26+00$ & $6.60+01$ & & & & & & \\
\hline-1.5 & $3.52+00$ & $3.46+00$ & $1.90+02$ & & & & & & \\
\hline-1.2 & $5.33+00$ & $5.29+00$ & $5.78+02$ & & & & & & \\
\hline-1.0 & $7.10+00$ & $7.08+00$ & $1.27+03$ & & & & & & \\
\hline-0.9 & $8.24+00$ & $8.22+00$ & $1.91+03$ & & & & & & \\
\hline-0.8 & $9.61+00$ & $9.59+00$ & $2.92+03$ & & & & & & \\
\hline-0.7 & $1.12+01$ & $1.12+01$ & $4.53+03$ & & & & & & \\
\hline-0.6 & $1.32+01$ & $1.32+01$ & $7.16+03$ & $9.11-01$ & $5.77-01$ & $6.23+00$ & & & \\
\hline-0.5 & $1.57+01$ & $1.57+01$ & $1.16+04$ & $1.25+00$ & $1.04+00$ & $1.09+01$ & & & \\
\hline-0.4 & $1.88+01$ & $1.88+01$ & $1.91+04$ & $1.71+00$ & $1.56+00$ & $2.17+01$ & & & \\
\hline-0.3 & $2.26+01$ & $2.26+01$ & $3.26+04$ & $2.30+00$ & $2.20+00$ & $4.54+01$ & & & \\
\hline-0.2 & $2.76+01$ & $2.76+01$ & $5.74+04$ & $3.10+00$ & $3.02+00$ & $9.72+01$ & & & \\
\hline-0.1 & $3.41+01$ & $3.41+01$ & $1.05+05$ & $4.18+00$ & $4.12+00$ & $2.14+02$ & & & \\
\hline 0.0 & $4.28+01$ & $4.28+01$ & $2.02+05$ & $5.67+00$ & $5.63+00$ & $4.88+02$ & $1.81+00$ & $1.43+00$ & $1.17+01$ \\
\hline 0.1 & $5.47+01$ & $5.47+01$ & $4.12+05$ & $7.79+00$ & $7.75+00$ & $1.16+03$ & $2.71+00$ & $2.49+00$ & $2.95+01$ \\
\hline 0.2 & $7.17+01$ & $7.17+01$ & $9.02+05$ & $1.09+01$ & $1.09+01$ & $2.95+03$ & $4.10+00$ & $3.96+00$ & $8.75+01$ \\
\hline 0.3 & $9.69+01$ & $9.69+01$ & $2.17+06$ & $1.56+01$ & $1.56+01$ & $8.11+03$ & $6.29+00$ & $6.20+00$ & $2.86+02$ \\
\hline 0.4 & $1.36+02$ & $1.36+02$ & $5.90+06$ & $2.31+01$ & $2.31+01$ & $2.50+04$ & $9.89+00$ & $9.83+00$ & $1.04+03$ \\
\hline 0.5 & $2.03+02$ & $2.03+02$ & $1.89+07$ & $3.62+01$ & $3.62+01$ & $9.02+04$ & $1.62+01$ & $1.62+01$ & $4.37+03$ \\
\hline 0.6 & $3.27+02$ & $3.27+02$ & $7.77+07$ & $6.12+01$ & $6.12+01$ & $4.12+05$ & $2.87+01$ & $2.87+01$ & $2.29+04$ \\
\hline 0.7 & $6.01+02$ & $6.01+02$ & $4.68+08$ & $1.17+02$ & $1.17+02$ & $2.75+06$ & $5.71+01$ & $5.71+01$ & $1.72+05$ \\
\hline 0.8 & $1.39+03$ & $1.39+03$ & $5.72+09$ & $2.83+02$ & $2.83+02$ & $3.69+07$ & $1.43+02$ & $1.43+02$ & $2.58+06$ \\
\hline 0.9 & $5.75+03$ & $5.75+03$ & $3.92+11$ & $1.21+03$ & $1.21+03$ & $2.77+09$ & $6.32+02$ & $6.32+02$ & $2.13+08$ \\
\hline 1.2 & $1.57+03$ & $1.57+03$ & $7.45+09$ & $3.67+02$ & $3.67+02$ & $6.70+07$ & $2.07+02$ & $2.07+02$ & $6.54+06$ \\
\hline 1.5 & $2.73+02$ & $2.73+02$ & $3.68+07$ & $6.98+01$ & $6.98+01$ & $4.07+05$ & $4.19+01$ & $4.19+01$ & $4.78+04$ \\
\hline 1.8 & $1.15+02$ & $1.15+02$ & $2.63+06$ & $3.19+01$ & $3.19+01$ & $3.48+04$ & $2.02+01$ & $2.02+01$ & $4.74+03$ \\
\hline 2.2 & $5.67+01$ & $5.67+01$ & $2.89+05$ & $1.71+01$ & $1.71+01$ & $4.72+03$ & $1.15+01$ & $1.15+01$ & $7.63+02$ \\
\hline 2.7 & $3.18+01$ & $3.18+01$ & $4.67+04$ & $1.06+01$ & $1.05+01$ & $9.56+02$ & $7.58+00$ & $7.45+00$ & $1.85+02$ \\
\hline 3.3 & $1.99+01$ & $1.98+01$ & $1.03+04$ & $7.28+00$ & $7.21+00$ & $2.65+02$ & $5.55+00$ & $5.34+00$ & $6.26+01$ \\
\hline 3.9 & $1.41+01$ & $1.41+01$ & $3.38+03$ & $5.63+00$ & $5.53+00$ & $1.07+02$ & $4.54+00$ & $4.24+00$ & $3.05+01$ \\
\hline 4.7 & $1.01+01$ & $1.01+01$ & $1.11+03$ & $4.44+00$ & $4.29+00$ & $4.47+01$ & $3.81+00$ & $3.38+00$ & $1.63+01$ \\
\hline 5.6 & $7.69+00$ & $7.64+00$ & $4.32+02$ & $3.69+00$ & $3.49+00$ & $2.21+01$ & $3.37+00$ & $2.79+00$ & $1.06+01$ \\
\hline 6.8 & $5.88+00$ & $5.81+00$ & $1.68+02$ & $3.12+00$ & $2.85+00$ & $1.15+01$ & $3.09+00$ & $2.26+00$ & $7.94+00$ \\
\hline 8.2 & $4.69+00$ & $4.60+00$ & $7.36+01$ & $2.76+00$ & $2.39+00$ & $6.94+00$ & $2.97+00$ & $1.81+00$ & $7.21+00$ \\
\hline 10.0 & $3.82+00$ & $3.68+00$ & $3.37+01$ & $2.50+00$ & $2.00+00$ & $4.65+00$ & $3.00+00$ & $1.33+00$ & $8.04+00$ \\
\hline 12.0 & $3.25+00$ & $3.07+00$ & $1.78+01$ & $2.37+00$ & $1.69+00$ & $3.67+00$ & $3.21+00$ & $8.07-01$ & $1.12+01$ \\
\hline 15.0 & $2.76+00$ & $2.51+00$ & $9.07+00$ & $2.30+00$ & $1.34+00$ & $3.30+00$ & $3.85+00$ & $-1.27-01$ & $2.57+01$ \\
\hline 18.0 & $2.48+00$ & $2.15+00$ & $5.72+00$ & $2.34+00$ & $1.05+00$ & $3.58+00$ & $5.12+00$ & $-1.59+00$ & $9.30+01$ \\
\hline 22.0 & $2.27+00$ & $1.83+00$ & $3.83+00$ & $2.50+00$ & $6.72-01$ & $4.80+00$ & $9.90+00$ & $-6.52+00$ & $1.66+03$ \\
\hline 33.0 & $2.09+00$ & $1.29+00$ & $2.51+00$ & $3.58+00$ & $-7.09-01$ & $2.33+01$ & & & \\
\hline
\end{tabular}


$V 2$ below which the lens behaves like a mirror. These values are $V 2 / V 1=-2.9$ for $x=0.2,-0.744$ for $x=0.5$ and -0.166 for $x=1$; the computation has been carried on to the vicinity of these values. From this table, it can be noticed that the lens convergence is zero for $V 2 / V 1=1$, and that it increases when $V 2$ decreases. When $V 2$ increases above 1 , the convergence increases too and reaches a peak. The system becomes afocal, then multiple foci appear. It can be noticed also that in weak lenses ( $F 1$ over 3 or 4 ), the principal planes coincide with each other and with the reference plane : weak lenses are also thin lenses. This table equally supports what had already been noticed by Adams and Read [3] and by Slodzian and Figueras [9] about the spherical aberration : for the same focal length, a unipotential lens within which the electrons are accelerated in the central part $(V 2>V 1)$ has a spherical aberration constant lower than that of a lens in which the electrons are decelerated $(V 2<V 1)$. For instance, if $x=0.5$ and $F 1=2.3$, it appears that the corresponding aberration can be either 3.3 or 45 . In a general way, the spherical aberration of the accelerating lens, compared to the decelerating one, is all the better as the lens is more convergent.

The figures in table I can be compared to those of
Read and Harting [10] who have computed unipotential lenses by means of the charge distribution method. In their book, the optical properties of the lens with $g=d=0.2$ are computed for $x=0.5$ and 1 . Read and Harting's values are always low compared with those we have here. In relative value, the differences are slight, a few tenths of a percent ; they may exceptionally reach $1 \%$. We have also compared our computed values with the results of the measurements made by Heddle and Kurepa [11]. In their paper, they give values of the $V 2 / V 1$ ratio such that an object and an image located in given places are conjugated by means of a unipotential lens. The difference between our values and their measured ones is about $10 \%$ or less for the $V 2 / V 1$ ratio. For example, figure $4 a$ in [11] shows that there is a conjugation, for the given locations, when the $V 2 / V 1$ ratio equals 0.32 or 3.1 . The computed ratios are 0.35 or 3.2 , therefore the fit with experiments can be considered as satisfactory.

Table II shows the optical constants of a unipotential lens in which $g=d=0.5$ for three $x$ values 0.5 , 1 and 2 . The remarks which have been made about the behaviour of the optical properties of the lens $g=0.2$ still apply to this lens.

The computation of the mechanically asymmetrical

Table II. - Values of the cardinal elements and of the spherical aberration constant as a function of the voltage ratio $V 2 / V 1$ for a unipotential lens $g=d=0.5$. The first three columns correspond to $x=0.5$, the following three to $x=1$ and the last three to $x=2$.

\begin{tabular}{|c|c|c|c|c|c|c|c|c|c|}
\hline$V 2 / V 1$ & $F 1$ & $Z F 1$ & $S P 1$ & $F 1$ & $Z F 1$ & $S P 1$ & $F 1$ & $Z F 1$ & $S P 1$ \\
\hline- & - & - & - & - & - & - & - & - & - \\
\hline-0.7 & $8.05-01$ & $3.18-01$ & $6.04+00$ & & & & & & \\
\hline-0.6 & $1.08+00$ & $8.05-01$ & $7.99+00$ & & & & & & \\
\hline-0.5 & $1.47+00$ & $1.28+00$ & $1.49+01$ & & & & & & \\
\hline-0.4 & $1.98+00$ & $1.84+00$ & $2.97+01$ & & & & & & \\
\hline-0.3 & $2.65+00$ & $2.55+00$ & $6.12+01$ & & & & & & \\
\hline-0.2 & $3.53+00$ & $3.46+00$ & $1.29+02$ & & & & & & \\
\hline-0.1 & $4.73+00$ & $4.67+00$ & $2.81+02$ & $1.31+00$ & $5.71-01$ & $8.44+00$ & & & \\
\hline 0.0 & $6.38+00$ & $6.33+00$ & $6.32+02$ & $1.90+00$ & $1.55+00$ & $1.32+01$ & & & \\
\hline 0.1 & $8.70+00$ & $8.67+00$ & $1.49+03$ & $2.86+00$ & $2.65+00$ & $3.41+01$ & $3.24+00$ & $1.24+00$ & $3.68+01$ \\
\hline 0.2 & $1.21+01$ & $1.21+01$ & $3.74+03$ & $4.32+00$ & $4.19+00$ & $1.01+02$ & $3.98+00$ & $3.15+00$ & $3.86+01$ \\
\hline 0.3 & $1.73+01$ & $1.73+01$ & $1.02+04$ & $6.62+00$ & $6.53+00$ & $3.28+02$ & $5.76+00$ & $5.32+00$ & $1.00+02$ \\
\hline 0.4 & $2.56+01$ & $2.56+01$ & $3.11+04$ & $1.04+01$ & $1.03+01$ & $1.18+03$ & $8.90+00$ & $8.64+00$ & $3.66+02$ \\
\hline 0.5 & $3.99+01$ & $3.99+01$ & $1.11+05$ & $1.71+01$ & $1.70+01$ & $4.91+03$ & $1.46+01$ & $1.44+01$ & $1.65+03$ \\
\hline 0.6 & $6.72+01$ & $6.72+01$ & $5.05+05$ & $3.01+01$ & $3.01+01$ & $2.55+04$ & $2.57+01$ & $2.56+01$ & $9.35+03$ \\
\hline 0.7 & $1.28+02$ & $1.28+02$ & $3.35+06$ & $5.99+01$ & $5.99+01$ & $1.90+05$ & $5.14+01$ & $5.14+01$ & $7.58+04$ \\
\hline 0.8 & $3.10+02$ & $3.10+02$ & $4.48+07$ & $1.50+02$ & $1.50+02$ & $2.83+06$ & $1.29+02$ & $1.29+02$ & $1.21+06$ \\
\hline 0.9 & $1.32+03$ & $1.32+03$ & $3.34+09$ & $6.62+02$ & $6.62+02$ & $2.33+08$ & $5.76+02$ & $5.76+02$ & $1.05+08$ \\
\hline 1.2 & $3.98+02$ & $3.98+02$ & $7.97+07$ & $2.16+02$ & $2.16+02$ & $7.07+06$ & $1.93+02$ & $1.93+02$ & $3.64+06$ \\
\hline 1.5 & $7.52+01$ & $7.52+01$ & $4.79+05$ & $4.37+01$ & $4.37+01$ & $5.13+04$ & $4.01+01$ & $4.00+01$ & $2.88+04$ \\
\hline 1.8 & $3.42+01$ & $3.42+01$ & $4.06+04$ & $2.10+01$ & $2.10+01$ & $5.06+03$ & $1.98+01$ & $1.97+01$ & $3.07+03$ \\
\hline 2.2 & $1.83+01$ & $1.83+01$ & $5.46+03$ & $1.20+01$ & $1.19+01$ & $8.09+02$ & $1.17+01$ & $1.14+01$ & $5.45+02$ \\
\hline 2.7 & $1.13+01$ & $1.12+01$ & $1.09+03$ & $7.85+00$ & $7.72+00$ & $1.95+02$ & $8.02+00$ & $7.57+00$ & $1.53+02$ \\
\hline 3.3 & $7.71+00$ & $7.64+00$ & $3.01+02$ & $5.73+00$ & $5.53+00$ & $6.55+01$ & $6.19+00$ & $5.46+00$ & $6.50+01$ \\
\hline 3.9 & $5.95+00$ & $5.85+00$ & $1.20+02$ & $4.67+00$ & $4.38+00$ & $3.17+01$ & $5.34+00$ & $4.28+00$ & $4.14+61$ \\
\hline 4.7 & $4.67+00$ & $4.52+00$ & $4.98+01$ & $3.91+00$ & $3.49+00$ & $1.68+01$ & $4.85+00$ & $3.25+00$ & $3.27+01$ \\
\hline 5.6 & $3.86+00$ & $3.67+00$ & $2.45+01$ & $3.46+00$ & $2.88+00$ & $1.09+01$ & $4.73+00$ & $2.37+00$ & $3.40+01$ \\
\hline 6.8 & $3.26+00$ & $2.99+00$ & $1.26+01$ & $3.16+00$ & $2.33+00$ & $8.08+00$ & $5.06+00$ & $1.25+00$ & $5.06+01$ \\
\hline 8.2 & $2.87+00$ & $2.50+00$ & $7.56+00$ & $3.02+00$ & $1.87+00$ & $7.27+00$ & $6.17+00$ & $-4.13-01$ & $1.30+02$ \\
\hline 10.0 & $2.59+00$ & $2.08+00$ & $5.03+00$ & $3.04+00$ & $1.39+00$ & $8.03+00$ & $1.03+01$ & $-5.01+00$ & $1.36+03$ \\
\hline 12.0 & $2.44+00$ & $1.76+00$ & $3.95+00$ & $3.24+00$ & $8.67-01$ & $1.10+01$ & $9.90+01$ & $-9.40+01$ & $1.79+07$ \\
\hline 15.0 & $2.37+00$ & $1.40+00$ & $3.53+00$ & $3.86+00$ & $-5.50-02$ & $2.48+01$ & & & \\
\hline 18.0 & $2.41+00$ & $1.10+00$ & $3.81+00$ & $5.08+00$ & $-1.47+00$ & $8.61+01$ & & & \\
\hline 22.0 & $2.56+00$ & $7.10-01$ & $5.10+00$ & $9.51+00$ & $-6.06+00$ & $1.36+03$ & & & \\
\hline
\end{tabular}


and electrically symmetrical lenses has enabled us to study the influence of the asymmetry rate on the optical properties. For these lenses, the following relations still hold : $F 1=F 2, Z F 1=Z F 2$ and $S P 1=S P 2$.

Figure 3 has been drawn for a lens in which $g$ is maintained at a constant value 0.2. Its shape is modified from $d=0.2$. The length $d$ of the right-hand gap is varied so that, either the distance $2 x$ between the median planes of the two gaps remains constant and equal to 0.4 , or the length of the inner cylinder $l=2 x-(g+d) / 2$ remains constant and equal to 0.2 . For $d=0.2$, the $F 1$ and $S P 1$ values are taken from table I. When $d$ varies to maintain $x$ constant, the representative points of $F 1$ and $S P 1$ are aligned on very slightly ascending straight lines (full curves). They are parallel to each other, so the relative varia-

Fig. 3. - Curves showing the variation of $F 1$ and $S P 1$ in the case of mechanical asymmetry for the lens $g=0.2$, with $d$ variable so that either $x=0.2$ (full line curves) or $l=0.2$ (broken curves). The curves concerning $F 1$ are plotted with circles and those concerning $S P 1$ are plotted with crosses. The ratio $V 2 / V 1$ equals -1.2 for curves $a,-0.5$ for $b, 0$ for $c, 0.5$ for $d, 0.9$ for $e$ and 15 for $f$.

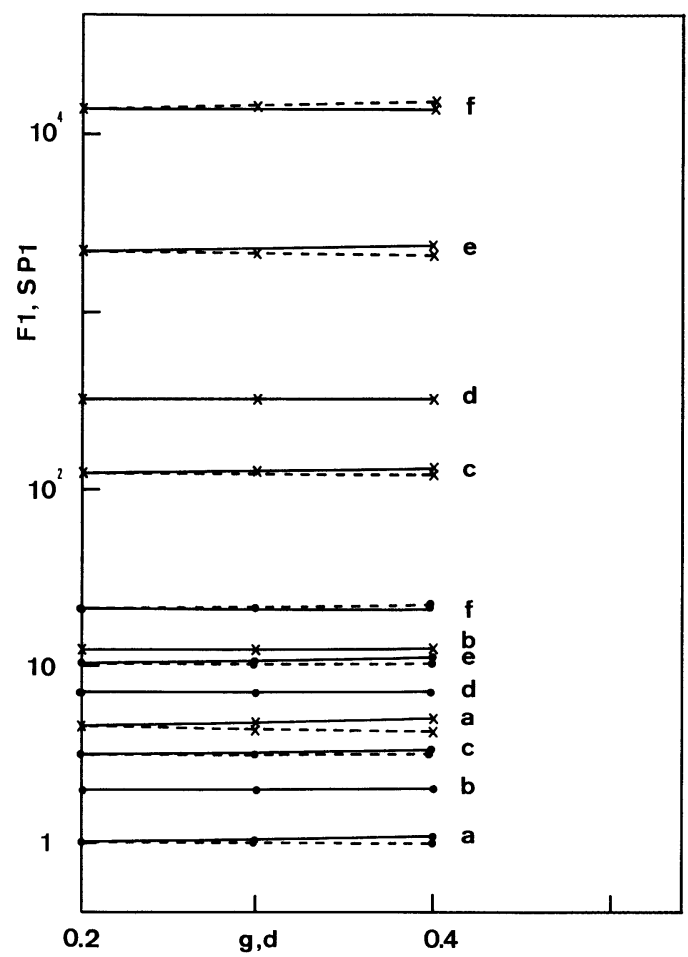

Table III. - The object focal length $F 1$ of the lens $g=d=x=0.2$ as a function of the ratios $V 2 / V 1$ and $V 3 / V 1$.

\begin{tabular}{|c|c|c|c|c|c|c|c|c|c|c|c|c|}
\hline$V 3 / V 1=$ & 1.2 & 1.5 & 1.8 & 2.2 & 2.7 & 3.3 & 39 & 4.7 & 5.6 & 6.8 & 8.2 & 10.0 \\
\hline$V 2 / V 1$ & & & & & & & & & & & & \\
\hline-10.0 & & & & & & & & & & & & $4.47-01$ \\
\hline-8.2 & & & & & & & & & & $|4.16-01|$ & D.J5 - & $6.66-$ \\
\hline-6.8 & & & & & & & & & $5.23-0$ & & & $8.50-01$ \\
\hline-5.6 & & & & & & & $5.16-01 \mid$ & $6.97-01$ & & $9.60+$ & & $1.01+00$ \\
\hline-4.7 & & & & & $4.84-01$ & $6.88-01$ & $8.69-01$ & $1.04+00$ & $1.14+00$ & $1.19+$ & $1.18+00$ & $1.12+00$ \\
\hline-3.9 & & & & $6.46-01)$ & $9.02-01$ & $1.14+00$ & $1.30+0$ & $1.41+00$ & $1.44+00$ & $1.40+$ & $1.32+$ & $1.21+00$ \\
\hline-3.3 & & $6.01-01$ & $8.31-01$ & $1.11+00 \mid$ & $1.39+00$ & $1.59+00$ & $1.69+00$ & $1.72+00$ & $1.67+00$ & $1.56+00$ & $1.43+0$ & $1.28+00$ \\
\hline-2.7 & $8.75-01 \mid$ & $1.22+00$ & $1.52+00$ & $|1.82+00|$ & $2.05+0$ & $2.16+00$ & $2.15+00$ & $2.06+00$ & $1.91+00$ & $1.72+c$ & $|1.53+00|$ & $1.34+00$ \\
\hline-2.2 & $68+00$ & $2.11+00$ & $2.43+00$ & $|2.68+00|$ & $2.79+0$ & $2.73+00$ & $2.58+00$ & $2.35+00 \mid$ & $2.11+00$ & $1.84+00 \mid$ & $|1.61+00|$ & $1.39+00$ \\
\hline-1.8 & $.76+00$ & $3.22+00$ & $3.49+00$ & $3.61+00 \mid$ & $3.51+00$ & $3.24+00 \mid$ & $2.94+00 \mid$ & $2.58+00$ & $2.26+00$ & $1.93+00$ & $1.66+00$ & $1.42+00$ \\
\hline-1.5 & $.99+00$ & $4.43+00$ & $4.58+00$ & $4.48+0$ & $4.13+0$ & $3.65+00$ & $|3.22+00|$ & $2.75+00$ & $2.37+00 \mid$ & $2.00+00$ & $1.70+00 \mid$ & $1.45+00$ \\
\hline-1.2 & $5.83+00$ & $6.12+00$ & $6.01+00$ & $5.34+00 \mid$ & $4.83+0$ & $4.08+00$ & $3.49+00$ & $2.92+00$ & $2.47+00$ & $2.06+00$ & $|1.74+00|$ & $1.47+00$ \\
\hline-1.0 & $.59+00$ & $7.64+00$ & $7.20+00$ & $6.35+00$ & $5.33+$ & $4.37+00$ & $3.68+00$ & $3.03+00$ & $2.54+00 \mid$ & $2.10+00$ & $1.77+00$ & $1.49+00$ \\
\hline-0.8 & $9.99+00$ & $9.58+00$ & $8.62+00$ & $|7.25+00|$ & $5.85+00$ & $4.66+00$ & $3.86+00$ & $3.13+00 \mid$ & $2.60+00$ & $2.13+00$ & $|1.79+00|$ & $1.50+00$ \\
\hline-0.6 & $.33+0$ & $1.21+01$ & $1.03+01$ & $8.24+0$ & $6.39+0$ & $4.95+00$ & $4.03+00$ & $3.23+00$ & $2.66+00$ & $2.17+00 \mid$ & $|1.81+00|$ & $1.51+00$ \\
\hline-0.4 & $1.82+0$ & $1.53+01$ & $1.23+01$ & $9.31+00$ & $6.93+00$ & $5.24+00$ & $4.20+00 \mid$ & $3.33+00 \mid$ & $2.72+00$ & $2.21+00$ & $1.83+00 \mid$ & $1.53+00$ \\
\hline-0.2 & $.55+01$ & $1.96+01$ & $1.46+01$ & $1.04+01 \mid$ & $7.48+0$ & $5.51+00$ & & $3.42+00 \mid$ & $2.77+00 \mid$ & $2.24+00 \mid$ & $1.85+00$ & $1.54+00$ \\
\hline 0.0 & $.68+01$ & $2.50+01$ & $1.73+01$ & $|1.16+01|$ & $8.02+00 \mid$ & $5.77+00$ & $4.51+00$ & $3.50+00$ & $2.82+00$ & $2.27+00 \mid$ & $|1.87+00|$ & $1.55+00$ \\
\hline 0.2 & $5.54+01$ & $3.20+01$ & $2.02+01$ & $|1.28+01|$ & $8.53+00$ & $6.02+00$ & $4.65+00$ & & $2.87+00 \mid$ & $2.30+00 \mid$ & $|1.89+00|$ & $1.56+00$ \\
\hline 0.4 & $.77+01$ & $4.07+01$ & $2.33+01$ & $1.40+01$ & $9.01+0$ & $6.25+00$ & $4.78+00$ & $3.65+00 \mid$ & $2.91+00$ & $|2.32+00|$ & $|1.90+00|$ & $1.57+00$ \\
\hline 0.6 & $.46+02$ & $5.05+01$ & $2.64+01$ & $1.50+01 \mid$ & $9.44+00$ & $6.45+00$ & $4.89+00$ & $3.72+00$ & $2.95+00$ & $2.35+00 \mid$ & $|1.92+00|$ & $1.58+00$ \\
\hline 0.8 & $.44+02$ & $6.04+01$ & $2.91+01$ & $1.59+01$ & $9.82+00$ & $6.63+00$ & $5.00+00$ & $3.78+00$ & $2.99+00 \mid$ & $2.37+00 \mid$ & $1.93+00$ & $1.59+00$ \\
\hline 1.0 & $.58+02$ & $6.85+01$ & $3.13+01$ & $1.67+01$ & $1.01+01$ & $6.78+00$ & $5.09+00$ & $3.83+00$ & $3.03+00 \mid$ & $2.39+00 \mid$ & $|1.95+00|$ & $1.60+00$ \\
\hline 1.2 & $.27+02$ & $7.18+01$ & $3.25+01$ & $|1.72+01|$ & $1.04+0$ & $6.90+00$ & $5.16+00$ & $3.88+00$ & $3.05+00$ & $2.41+00$ & $\mid 1.96+00$ & $1.61+00$ \\
\hline 1.5 & $.08+02$ & $6.62+01$ & $3.28+01$ & $1.76+01 \mid$ & $1.06+01$ & $7.04+00$ & $5.25+00$ & $3.93+00$ & $3.09+00 \mid$ & $2.43+00 \mid$ & $1.98+00 \mid$ & $1.62+00$ \\
\hline 1.8 & $.15+02$ & $5.80+01$ & $3.07+01$ & $1.74+01 \mid$ & $1.07+01$ & $7.11+00 \mid$ & $5.31+00$ & $3.97+00$ & $3.12+00$ & $2.45+00$ & $|1.99+00|$ & $1.63+00$ \\
\hline 2.2 & $.17+01$ & $4.37+01$ & $2.76+01$ & $1.65+01 \mid$ & $1.05+01$ & $7.12+00$ & $5.34+00$ & $4.01+00$ & $3.15+00 \mid$ & $2.47+00 \mid$ & $2.00+00 \mid$ & $1.64+00$ \\
\hline 2.7 & $|3.54+01|$ & $3.06+01$ & $2.27+01$ & $1.52+01 \mid$ & $1.01+01 \mid$ & $7.02+00 \mid$ & $5.32+00$ & $4.02+00$ & $3.17+00 \mid$ & $2.49+00$ & $2.02+00 \mid$ & $1.65+00$ \\
\hline 3.3 & $.20+01$ & $2.12+01 \mid$ & $1.77+01$ & $1.32+01 \mid$ & $9.42+00$ & $6.77+00$ & $5.23+00$ & $4.00+00$ & $3.17+00$ & $2.50+00 \mid$ & $2.03+00 \mid$ & $1.66+00$ \\
\hline 3.9 & $1.56+01 \mid$ & $1.57+01$ & $1.41+01$ & $|1.14+01|$ & $8.62+0$ & $6.45+00$ & $5.07+00 \mid$ & $3.94+00 \mid$ & $|3.15+00|$ & $2.50+00$ & $2.04+00 \mid$ & $1.67+00$ \\
\hline 4.7 & $1.10+01 \mid$ & $1.14+01$ & $1.08+01$ & $9.38+00 \mid$ & $7.60+00 \mid$ & $5.97+00$ & $4.84+00$ & $3.82+00$ & $3.10+00$ & $2.48+00$ & $2.03+00$ & $1.67+00$ \\
\hline 5.6 & $|8.30+00|$ & $8.65+00$ & $8.45+00$ & $|7.72+00|$ & $6.60+0$ & $5.43+00 \mid$ & $|4.53+00|$ & $|3.68+00|$ & $3.02+00 \mid$ & $2.45+00 \mid$ & $|2.02+00|$ & $1.67+00$ \\
\hline 6.8 & $6.26+00$ & $6.55+00$ & $6.52+00$ & $6.19+00$ & $5.56+00$ & $4.79+00$ & $4.13+00$ & $3.45+00$ & $2.90+00$ & $2.39+00 \mid$ & $|1.99+00|$ & $1.66+00$ \\
\hline 8.2 & $4.94+00 \mid$ & $5.14+00 \mid$ & $5.17+00 \mid$ & $5.02+00 \mid$ & $4.66+00 \mid$ & $4.17+00$ & $3.71+00 \mid$ & $|3.19+00|$ & $2.74+00 \mid$ & $2.30+00$ & $|1.94+00|$ & $1.63+00$ \\
\hline 10.0 & $|3.97+00|$ & $4.10+00 \mid$ & $4.13+00$ & $|4.06+00|$ & $|3.87+00|$ & $|3.57+00|$ & $|3.27+00|$ & $|2.89+00|$ & $|2.55+00|$ & $2.19+00 \mid$ & $1.88+00$ & $1.60+00$ \\
\hline
\end{tabular}


tions of the optical constants do not depend on the $V 2 / V 1$ ratio. On the contrary, when $d$ varies to maintain $l$ constant, the representative points are aligned on clearly descending straight lines. Their slopes vary with the $V 2 / V 1$ ratio : they decrease when $V 2 / V 1$ increases.

Figure 4 is drawn for $g=0.2$ and $d$ varies to maintain either $x=0.5$ or $l=0.8$. The shapes of these curves are very similar to those in figure 3 . It can be noticed again that the optical elements remain practically constant when $d$ varies with $x$ constant, while they decrease when $l$ is constant.

It can be deduced from these curves that the important geometrical parameter, which determines the optical properties of an electrically symmetrical lens for a given $V 2 / V 1$ ratio, is the $2 x$ distance. If constraints of building imply that a lens with a given focal

Fig. 4. - Curves showing the variation of $F 1$ and $S P 1$ in the case of mechanical asymmetry for the lens $g=0.2$, with $d$ variable so that either $x=0.5$ (full line curves) or $l=0.5$ (broken curves). The curves concerning $F 1$ are plotted with circles and those concerning $S P 1$ are plotted with crosses. The ratio $V 2 / V 1$ equals -0.5 for curves $a, 0$ for $b, 0.5$ for $c, 0.9$ for $d$ and 15 for $e$.

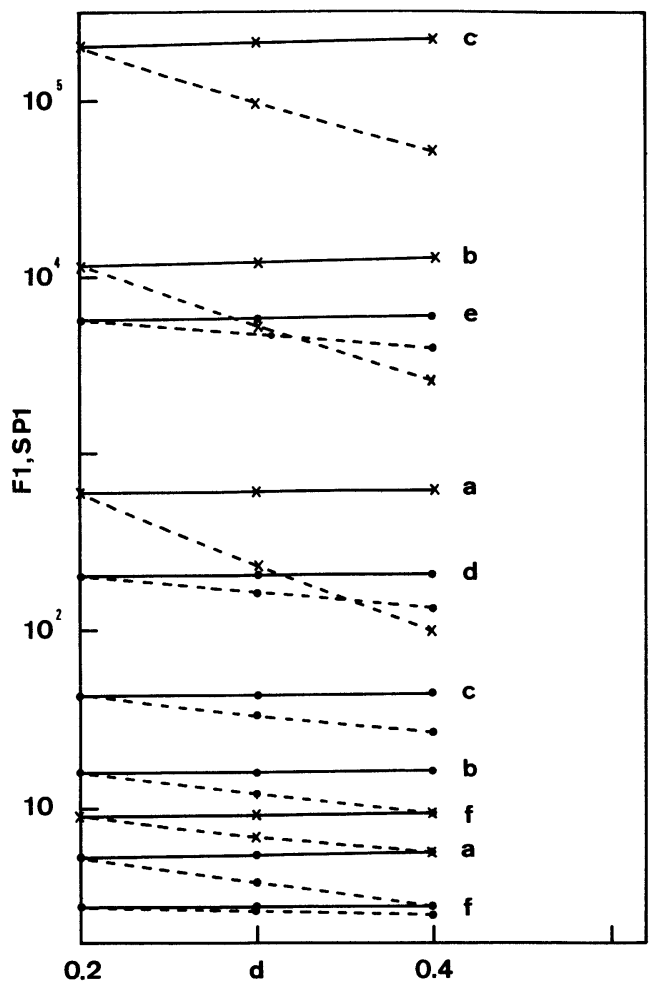

Table IV. - The position ZF1 of the object focus of the same lens as a function of the ratios $V 2 / V 1$ and $V 3 / V 1$.

\begin{tabular}{|c|c|c|c|c|c|c|c|c|c|c|c|c|}
\hline$V 3 / V 1=$ & 1.2 & 1.5 & 1.8 & 2.2 & 2.7 & 3.3 & 3.9 & 4.7 & 5.6 & 6.8 & 8.2 & 10.0 \\
\hline$V 2 / V 1$ & & & & & & & & & & & & \\
\hline $\begin{array}{r}-10.0 \\
-8 .\end{array}$ & & & & & & & & & & & & $\left|\begin{array}{l}7.08-01 \\
11+00\end{array}\right|$ \\
\hline $\begin{array}{l}-8.2 \\
-6.8\end{array}$ & & & & & & & & & $6.57-01$ & $\left|\begin{array}{ll}3.01 & -01 \\
9.81 & -01\end{array}\right|$ & & $\mid \begin{array}{l}1.11+00 \\
1.43+00\end{array}$ \\
\hline-5.6 & & & & & & & $5.19-01$ & $8.81-01$ & $1.17+00$ & & & \\
\hline-4.7 & & & & & $|2.82-01|$ & $|7.52-01|$ & $1.07+00$ & $1.37+00$ & $1.69+$ & $1.79+$ & 1.90 & $1.94+00$ \\
\hline-3.9 & & & & $5.59-01$ & $\mid 9.87-01$ & $|1.37+00|$ & $1.65+00$ & $1.90+00$ & $2.05+00$ & $2.14+00$ & $2.16+00$ & $2.13+00$ \\
\hline-3.3 & & $3.39-01$ & $|7.67-01|$ & $1.19+00$ & $1.60+00$ & $|1.96+00|$ & $2.19+00$ & $2.35+00$ & $|2.41+00|$ & $2.41+00$ & $2.35+0$ & $2.26+00$ \\
\hline-2.7 & $7.05-01$ & $1.20+00$ & $|1.60+00|$ & $2.05+00$ & $2.43+00$ & $2.70+00 \mid$ & $2.82+00$ & $2.84+00$ & $|2.79+00|$ & $2.67+00$ & $2.54+$ & $2.39+00$ \\
\hline-2.2 & $1.64+00$ & $2.20+00$ & $|2.64+00|$ & $3.06+$ & $3.34+00 \mid$ & $3.45+$ & $3.41+$ & $3.28+$ & $3.10+$ & $2.89+00 \mid$ & $|2.69+00|$ & $2.50+00$ \\
\hline-1.8 & $2.78+00$ & $3.42+$ & 3.85 & $4.16+$ & $4.25+$ & $4.13+$ & $3.92+$ & $3.63+00$ & $3.35+00 \mid$ & $3.06+00$ & & $2.58+00$ \\
\hline-1.5 & $4.08+00$ & $4.73+00$ & $5.09+00 \mid$ & $\{5.20+00$ & $5.04+00 \mid$ & $4.68+00$ & $4.32+00$ & $3.90+00$ & $3.54+00$ & $3.18+00$ & $2.89+00$ & $2.64+00$ \\
\hline-1.2 & $6.00+00$ & $6.59+00$ & $6.71+00$ & $6.46+00$ & $5.92+00$ & $5.26+00 \mid$ & $4.72+00$ & $4.16+00$ & $|3.72+00|$ & $3.30+00$ & $2.97+00$ & $2.69+00$ \\
\hline-1.0 & $83+00$ & $8.24+c$ & $|8.07+00|$ & $7.44+c$ & $6.55+00$ & $|5.66+00|$ & $4.99+0$ & $4.34+00$ & $|3.83+00|$ & $|3.38+00|$ & $|3.02+00|$ & $2.73+00$ \\
\hline-0.8 & $1.03+c$ & $1.04+$ & $9.70+0$ & $8.53+0$ & $7.23+$ & $6.07+$ & $5.25+$ & $4.50+00$ & $3.94+0$ & $|3.45+00|$ & $|3.07+00|$ & $|2.76+00|$ \\
\hline-0.6 & $1.38+c$ & $1.31+$ & $1.16+$ & $9.73+$ & $7.92+$ & $6.47+$ & $51+$ & $4.67+00$ & $4.05+$ & $|3.52+00|$ & $|3.12+00|$ & $|2.80+00|$ \\
\hline-0.4 & $1.89+($ & $1.67+$ & $1.39+$ & $1.10-$ & $8.64+$ & $6.87-$ & $5.77+$ & $4.83+00 \mid$ & $|4.16+00|$ & $3.59+0$ & $|3.17+00|$ & $|2.83+00|$ \\
\hline-0.2 & $2.65+01$ & $2.13+01$ & $|1.66+01|$ & $1.24+01$ & $9.36+00$ & $7.26+00 \mid$ & $6.01+00$ & $4.98+00$ & $|4.26+00|$ & $3.65+00 \mid$ & $|3.22+00|$ & $2.86+00$ \\
\hline 0.0 & $3.83+01$ & $2.74+01$ & $|1.97+01|$ & $1.39+01$ & $1.01+01$ & $7.64+00$ & $6.24+00$ & $5.12+00$ & $4.35+00$ & $3.72+00$ & $3.26+00$ & $2.89+00$ \\
\hline 0.2 & $5.77+01$ & $3.12+01$ & $|2.31+01|$ & $1.54+01$ & $1.07+01$ & $8.00+00 \mid$ & $6.46+00$ & $|5.26+00|$ & $|4.44+00|$ & $|3.78+00|$ & $|3.30+00|$ & $2.92+00$ \\
\hline 0.4 & $9.14+01$ & $4.46+01$ & $|2.67+01|$ & $1.68+01$ & $1.14+$ & $8.33+$ & $6.67+00$ & $|5.39+00|$ & $|4.53+00|$ & $3.84+00 \mid$ & $|3.34+00|$ & $2.95+00$ \\
\hline 0.6 & $1.52+$ & $5.56+c$ & $|3.03+01|$ & $1.81+$ & $1.20+$ & $8.64+$ & $6.86+00$ & $5.50+$ & $4.61+$ & $3.89+0$ & $|3.38+00|$ & $|2.98+00|$ \\
\hline 0.8 & $55+c$ & $6.66+$ & $3.35+$ & $93+$ & $1.25+$ & $8.91-$ & $33+$ & $5.61+$ & $4.68+$ & & $42+00$ & $|3.00+00|$ \\
\hline 1.0 & $3.75+c$ & $7.57+$ & $|3.62+01|$ & $2.03+$ & $1.30+$ & $9.16+$ & $\mid 7.20+00$ & $5.72+$ & $4.76+$ & )$|3.99+00|$ & $|3.46+00|$ & $|3.03+00|$ \\
\hline 1.2 & $3.43+02$ & $7.95+01$ & $|3.77+01|$ & $2.10+01$ & $1.33+01$ & $9.35+00$ & $7.32+00$ & $|5.81+00|$ & $|4.82+00|$ & $|4.04+00|$ & $|3.49+00|$ & $3.06+00$ \\
\hline 1.5 & $2.19+02$ & $7.36+01$ & $|3.81+01|$ & $2.15+01$ & $1.37+01 \mid$ & $|9.59+00|$ & $7.49+00$ & $|5.92+00|$ & $|4.91+00|$ & $4.10+00$ & $|3.53+00|$ & $3.09+00$ \\
\hline 1.8 & $1.21+02$ & $6.47+01$ & $|3.59+01|$ & $2.15+01$ & $1.38+01 \mid$ & $9.75+00$ & $7.62+00$ & $6.02+00 \mid$ & $|4.98+00|$ & $|4.16+00|$ & $|3.58+00|$ & $3.12+00$ \\
\hline 2.2 & $6.50+01$ & $4.89+01$ & $|3.24+01|$ & $2.05+01$ & $|1.38+01|$ & $9.84+00 \mid$ & $|7.73+00|$ & $6.12+00 \mid$ & $|5.06+00|$ & $|4.22+00|$ & $|3.63+00|$ & $3.17+00$ \\
\hline 2.7 & $3.73+01$ & $3.44+0$ & $|2.68+01|$ & $1.90+$ & $1.33+$ & $9.79+$ & $7.78+00$ & $|6.20+00|$ & $|5.14+00|$ & $|4.29+00|$ & $|3.69+00|$ & $3.21+00 \mid$ \\
\hline 3.3 & $2.33+01$ & $|2.39+01|$ & $|2.10+01|$ & $1.66+01$ & $|1.25+01|$ & $9.54+00 \mid$ & $7.73+00 \mid$ & $|6.23+00|$ & $|5.20+00|$ & $|4.35+00|$ & $|3.75+00|$ & $3.26+00 \mid$ \\
\hline 3.9 & $1.65+01$ & $|1.78+01|$ & $|1.68+01|$ & $1.44+$ & $\mid 1.16+01$ & $9.19+$ & $7.57+00$ & $6.21+00$ & $|5.22+00|$ & $4.39+$ & $|3.79+00|$ & $3.30+00$ \\
\hline 4.7 & $17+01$ & $1.30+c$ & $|1.30+01|$ & $1.20+$ & $1.03+$ & $8.61+$ & $7.32+$ & $6.10+$ & $5.21+$ & $4.42+$ & $3.83+$ & $3.35+00 \mid$ \\
\hline 5.6 & $82+00$ & $\mid 9.92+00$ & $|1.03+01|$ & $9.99+$ & $9.09+$ & $7.93+$ & $6.95+$ & $5.95+$ & $|5.15+00|$ & $4.42+$ & $3.86+$ & $3.38+00$ \\
\hline 6.8 & $6.65+00$ & $7.54+00 \mid$ & $8.00+00 \mid$ & $8.10+00$ & $7.76+c$ & $7.10+$ & $6.44+00 \mid$ & $|5.69+00|$ & $|5.03+00|$ & $4.39+0$ & $|3.87+00|$ & $3.42+00 \mid$ \\
\hline 8.2 & $5.23+00$ & & $|6.39+00|$ & $6.64+00$ & $6.60+00 \mid$ & $|6.29+00|$ & $5.89+00$ & $|5.36+00|$ & $|4.85+00|$ & $|4.32+00|$ & $|3.85+00|$ & $|3.43+00|$ \\
\hline 10.0 & $4.17+00$ & $|4.74+00|$ & $5.13+00$ & $5.44+00$ & $|5.56+00|$ & $|5.48+00|$ & $5.29+00$ & $|4.96+00|$ & $|4.60+00|$ & $|4.19+00|$ & $|3.80+00|$ & $3.43+00 \mid$ \\
\hline
\end{tabular}


length should be mechanically asymmetrical, this must be achieved by altering the gap lengths so that $x$ remains constant. So, and for a first approximation, the optical constants remain those of the unipotential lens with the same $x$ and $V 2 / V 1$. For a second approximation, the optical constants of the unipotential lens have to be multiplied by a corrective factor slightly over 1 , proportional to $d-g$ and not depending on $V 2 / V 1$.

These curves also show that it is possible to build more convergent lenses than the unipotential lens with the same $V 2 / V 1$ and having a weaker spherical aberration. To achieve this, the shape of the lens must be altered while the length of the central cylinder is maintained constant. Figures 3 and 4 show that $F 1$ and $S P 1$ can sometimes decrease significantly and that even the $S P 1 / F 1$ ratio can be improved in that process.

6. Optical properties of electrically asymmetrical lenses. - We have computed these properties for electrons going from the region with potential $V 1$ to the region with potential $V 3$ and for lenses accelerating as a whole $(V 3>V 1)$.

We have computed the optical constants of a mechanically symmetrical lens in which $g=d=0.2$, for various values of the ratios $V 2 / V 1$ and $V 3 / V 1$. The results are shown in tables III to VII. Table III gives the object focal length $F 1$, and the image focal length $F 2$ can be deduced from it by

$$
F 2=F 1(V 3 / V 1)^{1 / 2} \text {. }
$$

Table IV gives the position $Z F 1$ of the object focus and table $\mathrm{V}$ the position $Z F 2$ of the image focus. Table VI gives the spherical aberration constant $S P 1$ for an image located to infinity and table VII the limit of the product $C_{\mathrm{s}} M^{4}$ for an object located to infinity. These elements vary in the same way with respect to the variations of $V 2 / V 1$ and $V 3 / V 1$. For a given $V 3 / V 1$ ratio, when $V 2 / V 1$ increases, each element increases, reaches a maximum and then decreases. When $V 3 / V 1$ is approximately equal to 1 , the maximum is very noticeable and obtained for a $V 2 / V 1$ ratio approximately equal to 1 . When $V 3 / V 1$ increases, the maximum gets less and less important and it is obtained for higher and higher $V 2 / V 1$ ratios. When $V 3 / V 1$ equals 10 , the lens is strong for all the values of $V 2 / V 1$, and its optical properties remain fairly similar to those of the two-cylinder lens with the same potential ratio, whatever the values of $V 2 / V 1$.

Table V. - The position ZF2 of the image focus of the same lens as a function of the ratios $V 2 / V 1$ and $V 3 / V 1$.

\begin{tabular}{|c|c|c|c|c|c|c|c|c|c|c|c|c|}
\hline$V 3 / V 1=$ & 1.2 & 1.5 & 1.8 & 2.2 & 2.7 & 3.3 & 3.9 & 4.7 & 5.6 & 6.8 & 8.2 & 10.0 \\
\hline$V 2 / V 1$ & & & & & & & & & & & & \\
\hline-10.0 & & & & & & & & & & & & $5.76-01$ \\
\hline-8.2 & & & & & & & & & & $|3.06-01|$ & $8.02-$ & $11.13+00$ \\
\hline-6.8 & & & & & & & & & $5.81-01$ & $1.03+0$ & & $0 \mid 1.50+00$ \\
\hline-5.6 & & & & & & & $4.19-01$ & $1|9.17-01|$ & $1 \mid 1.28+00$ & $0 \mid 1.58+$ & $0 \mid 1.74+$ & $01.79+00$ \\
\hline-4.7 & & & & & $|1.36-01|$ & $|7.57-01|$ & $1.17+00$ & $1.55+00$ & $01.82+00$ & $0 \mid 1.99+$ & $2.03+00$ & $01.97+00$ \\
\hline-3.9 & & & & $5.24-01$ & $1.06+00 \mid$ & $\mid 1.54+00$ & $1.88+c$ & & $02.31+00$ & & & $02.12+00$ \\
\hline-3.3 & & $|2.95-01|$ & $7.80-01 \mid$ & $1.29+00$ & $1.80+00$ & $2.24+00$ & $2.50+00$ & $02.66+00 \mid$ & $2.68+00$ & $02.59+00$ & $2.43+00$ & $02.21+00$ \\
\hline-2.7 & $7.07-01$ & $|1.25+00|$ & $1.73+00 \mid$ & $2.27+00$ & $2.75+00$ & $3.07+00 \mid$ & $3.19+00$ & $3.17+00$ & $3.05+00$ & $0 \mid 2.83+00$ & $2.57+00$ & $02.30+00$ \\
\hline-2.2 & $1.68+00$ & $|2.33+00|$ & $2.87+00 \mid$ & $3.39+00$ & $3.75+00 \mid$ & $3.87+00 \mid$ & $3.80+00$ & $3.60+00$ & $3.33+00$ & $03.00+00$ & $2.68+00$ & $0 \mid 2.36+00$ \\
\hline-1.8 & $2.86+00$ & $|3.62+00|$ & $4.17+00 \mid$ & $4.58+00$ & $0|4.71+00|$ & $4.57+00$ & $04.31+00$ & $0|3.83+00|$ & $03.55+00$ & $0|3.13+00|$ & $2.76+00$ & $02.40+00$ \\
\hline-1.5 & $4.19+00$ & $|5.00+00|$ & $5.47+00 \mid$ & $5.68+00$ & $|5.53+00|$ & $|5.13+00|$ & $4.70+00$ & $4.18+00$ & $3.70+00$ & $0 \mid 3.22+$ & $2.81+00$ & $02.43+00$ \\
\hline-1.2 & $6.15+00$ & $6.92+00 \mid$ & $7.17+00 \mid$ & $6.99+00$ & $|6.44+00|$ & $|5.71+00|$ & $5.08+00$ & $4.41+00$ & $3.84+00$ & $0|3.30+00|$ & $2.86+00$ & $02.45+00$ \\
\hline-1.0 & $8.01+00$ & $|8.63+00|$ & $8.58+00$ & $8.00+00$ & $|7.08+00|$ & $|6.10+00|$ & $05.32+00$ & $04.55+00$ & $03.93+00$ & $0|3.35+00|$ & $02.88+00$ & $02.47+00$ \\
\hline-0.8 & $1.05+01$ & $|1.08+01|$ & $|1.03+01|$ & $9.11+00$ & $7.75+00$ & $6.48+00 \mid$ & $5.56+00$ & $4.69+00$ & $04.02+00$ & $0|3.40+00|$ & $2.91+00$ & $02.48+00$ \\
\hline-0.6 & $1.41+01$ & $|1.36+01|$ & $|1.22+01|$ & $1.03+01$ & $1|8.43+00|$ & $6.86+00 \mid$ & $05.79+00$ & $4.82+00$ & $04.09+00$ & $0|3.44+00|$ & $2.93+00$ & $02.50+00$ \\
\hline-0.4 & $1.92+01$ & $|1.72+01|$ & $1.46+01 \mid$ & $1.16+01$ & $|9.13+00|$ & $7.23+$ & $06.01+00$ & $04.95+00$ & $04.16+00$ & $0|3.48+00|$ & $2.96+$ & $02.51+00$ \\
\hline-0.2 & $2.68+01$ & $|2.20+01|$ & $|1.73+01|$ & $1.30+01$ & $19.81+$ & 7.58 & $6.21+$ & $5.06+$ & $04.23+00$ & $0|3.51+00|$ & $2.98+$ & $02.52+00$ \\
\hline 0.0 & $3.88+01$ & $2.81+01$ & $2.04+01$ & $1.44+01$ & $1|1.05+01|$ & $7.91+00$ & $06.40+00$ & $0[5.16+00 \mid$ & $04.29+00$ & $03.55+00 \mid$ & $2.99+00$ & $02.53+00$ \\
\hline 0.2 & $5.83+01$ & $|3.58+01|$ & $|2.38+01|$ & $1.59+01$ & $|1.11+01|$ & $|8.21+00|$ & $6.57+00$ & $5.26+00$ & $04.34+00$ & $0|3.58+00|$ & $03.01+00$ & $02.54+00$ \\
\hline 0.4 & $9.21+01$ & $|4.34+01|$ & $|2.73+01|$ & $1.72+01$ & $1|1.17+01|$ & $|8.49+00|$ & $6.73+00$ & $5.34+00$ & $04.39+00$ & $03.61+00$ & $3.03+00$ & $02.54+00$ \\
\hline 0.6 & $1.53+02$ & $|5.62+01|$ & $3.08+01 \mid$ & $1.85+01$ & $|1.22+01|$ & $|8.73+00|$ & $6.86+00$ & $5.41+00 \mid$ & $4.43+00$ & $0|3.63+00|$ & $3.04+00$ & $02.55+00$ \\
\hline 0.8 & $2.56+02$ & $|6.71+01|$ & $3.39+01 \mid$ & $1.95+01$ & $|1.26+01|$ & $8.93+00 \mid$ & $6.98+00$ & $|5.48+00|$ & $0 \mid 4.47+00$ & $0|3.65+00|$ & $3.05+00$ & $02.55+00$ \\
\hline 1.0 & $3.75+02$ & $|7.59+01|$ & $|3.63+01|$ & $2.04+01$ & $|1.30+01|$ & $|9.11+00|$ & $7.08+00$ & $5.53+00 \mid$ & $04.50+00$ & $03.66+00$ & $03.06+00$ & $02.56+00$ \\
\hline 1.2 & $3.43+02$ & $7.94+$ & $3.77+01$ & $2.09+01$ & $|1.32+01|$ & $9.23+$ & $7.15+00$ & $|5.57+00|$ & $04.53+00$ & $0|3.68+00|$ & $3.06+00$ & $02.56+00$ \\
\hline 1.5 & $2.18+02$ & $|7.31+01|$ & $|3.78+01|$ & $2.13+01$ & $1.34+01 \mid$ & $9.35+00$ & $7.23+00$ & $5.62+00$ & $4.55+00$ & $0|3.69+00|$ & $3.07+00$ & $02.56+00$ \\
\hline 1.8 & $1.20+02$ & $26.38+01$ & $3.53+01 \mid$ & $2.10+01$ & $1.35+01 \mid$ & $|9.39+00|$ & $7.26+00$ & $5.64+00$ & $4.57+00$ & $0|3.70+00|$ & $3.07+00$ & $02.56+00$ \\
\hline 2.2 & $6.42+01$ & $4.78+01 \mid$ & $3.15+01 \mid$ & $1.98+01$ & $|1.32+01|$ & $|9.34+00|$ & $7.25+00$ & $5.64+00$ & $04.57+00$ & $0|3.70+00|$ & $3.07+00$ & $02.56+00$ \\
\hline 2.7 & $3.67+01$ & $13.33+01$ & $2.57+01$ & $1.80+01$ & $|1.25+01|$ & $|9.12+00|$ & $7.15+00$ & $5.60+00 \mid$ & $04.55+00$ & $03.69+00$ & $3.07+00$ & $02.55+00$ \\
\hline 3.3 & $2.28+01$ & $2.29+01 \mid$ & $|1.99+01|$ & $1.55+01$ & $1|1.16+01|$ & $|8.69+00|$ & $06.95+00$ & $5.50+00$ & $04.50+00$ & $0|3.66+00|$ & $3.05+00$ & $02.54+00$ \\
\hline 3.9 & $1.61+01$ & $1|1.69+01|$ & $1.57+01$ & $1.32+01$ & $|1.05+01|$ & $|8.19+00|$ & $6.65+00$ & $05.36+00$ & $04.42+00$ & $0|3.61+00|$ & $03.02+00$ & $02.52+00$ \\
\hline 4.7 & $1.13+01$ & $1.22+01$ & $|1.20+01|$ & $1.08+01$ & $|9.13+00|$ & $7.46+00$ & $6.25+00$ & $5.12+00$ & $4.28+00$ & $03.53+00$ & $2.97+00$ & $02.49+00$ \\
\hline 5.6 & $8.48+00$ & $9.17+00$ & $|9.26+00|$ & $8.78+00$ & $|7.82+00|$ & $6.69+00 \mid$ & $5.76+00$ & $4.84+00$ & $04.09+00$ & $03.43+00$ & $2.90+00$ & $02.44+00$ \\
\hline 6.8 & $6.35+00$ & $06.86+00$ & $|7.05+00|$ & $6.92+00$ & $|6.46+00|$ & $|5.77+00|$ & $5.14+$ & $4.44+00$ & $03.84+00$ & $03.26+$ & $2.79+$ & $02.37+00$ \\
\hline 8.2 & $4.95+00$ & $5.31+00 \mid$ & $5.49+00 \mid$ & $6.51+00$ & $5.30+00 \mid$ & $4.92+00 \mid$ & $4.51+00$ & $4.01+00$ & $3.54+00$ & $0|3.07+00|$ & $2.65+00$ & $02.28+00$ \\
\hline 10.0 & $3.91+00$ & $4.15+00$ & $|4.29+00|$ & $4.36+00$ & $|4.29+00|$ & $|4.09+00|$ & $03.85+00$ & $3.52+00 \mid$ & $03.18+00$ & $0 \mid 2.82+00$ & $2.48+00$ & $02.16+00$ \\
\hline
\end{tabular}


The optical constants of the lenses decelerating as a whole $(V 3<V 1)$ can be obtained in the following way : as entry parameters of the tables, the $V 2 / V 1$ ratio of the accelerating lens is replaced by $V 2 / V 3$ and $V 3 / V 1$ by $V 1 / V 3$. Therefore, table III gives $F 1$, table IV $Z F 2$ and table $\mathrm{V} Z F 1 . S P 1$ is obtained from table VII by $S P 1_{\text {decel }}=S P 2_{\text {accel }}(V 1 / V 3)^{3 / 2}$ and $S P 2$ from table VI by $S P 2_{\text {decel }}=S P 1_{\text {accel }}(V 1 / V 3)^{3 / 2}$.

We have also computed the optical constants of the lens $g=d=0.2$ for two other values $x=1$ and $x=2$. They had also been calculated by Read by means of his method of charge distribution and published in his book [10]. When we compare his values to ours, we notice, as we had already seen for

Fig. 5. - Curves showing the variations of $F 1$ and $S P 1$ in the case of mechanical asymmetry for electrically asymmetrical lenses with $x=0.2$ and either $d=0.2$ and $g$ variable (full line curves), or $g=0.2$ and $d$ variable (broken curves). When the curves are confounded, the full line one only is plotted. The circles correspond to $F 1$ and the crosses to $S P 1$. The lenses plotted are the following : curves $a: V 2 / V 1=-5.6, V 3 / V 1=10 ;$ curves $b: V 2 / V 1=2.2$, $V 3 / V 1=8.2$; curves $c: V 2 / V 1=-0.6, V 3 / V 1=3.9$; curves $d$ : $V 2 / V 1=1.5, \quad V 3 / V 1=3.3 ;$ curves $e \quad: \quad V 2 / V 1=-0.2$, $V 3 / V 1=2.2$; curves $f: V 2 / V 1=3.3, V 3 / V 1=1.5$.

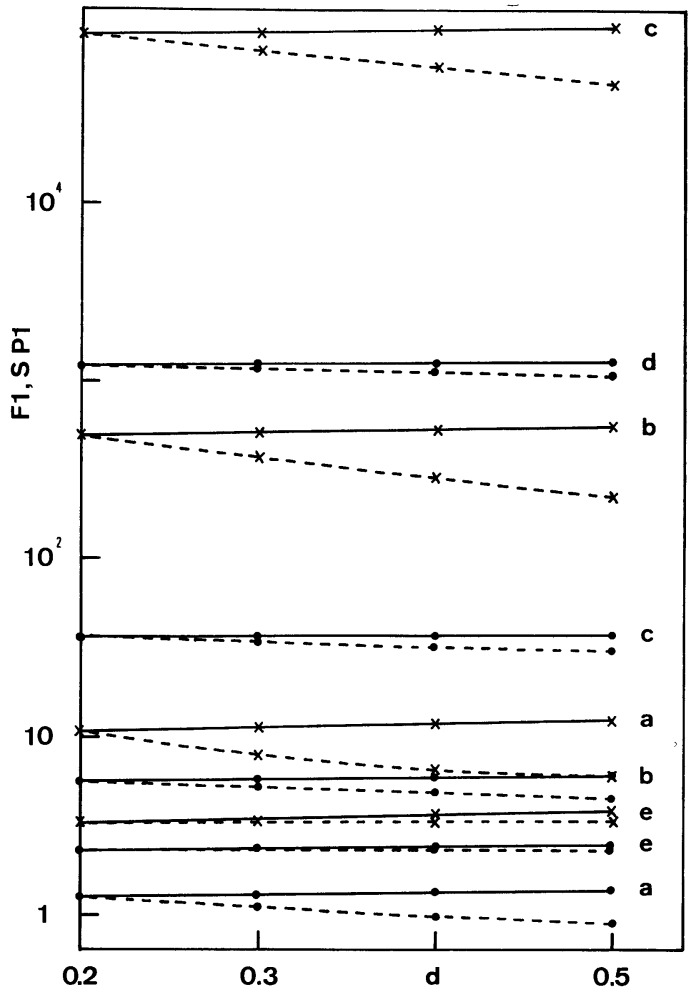

Table VI. - The spherical aberration constant SP1 of the same lens as a function of the ratios $V 2 / V 1$ and $V 3 / V 1$.

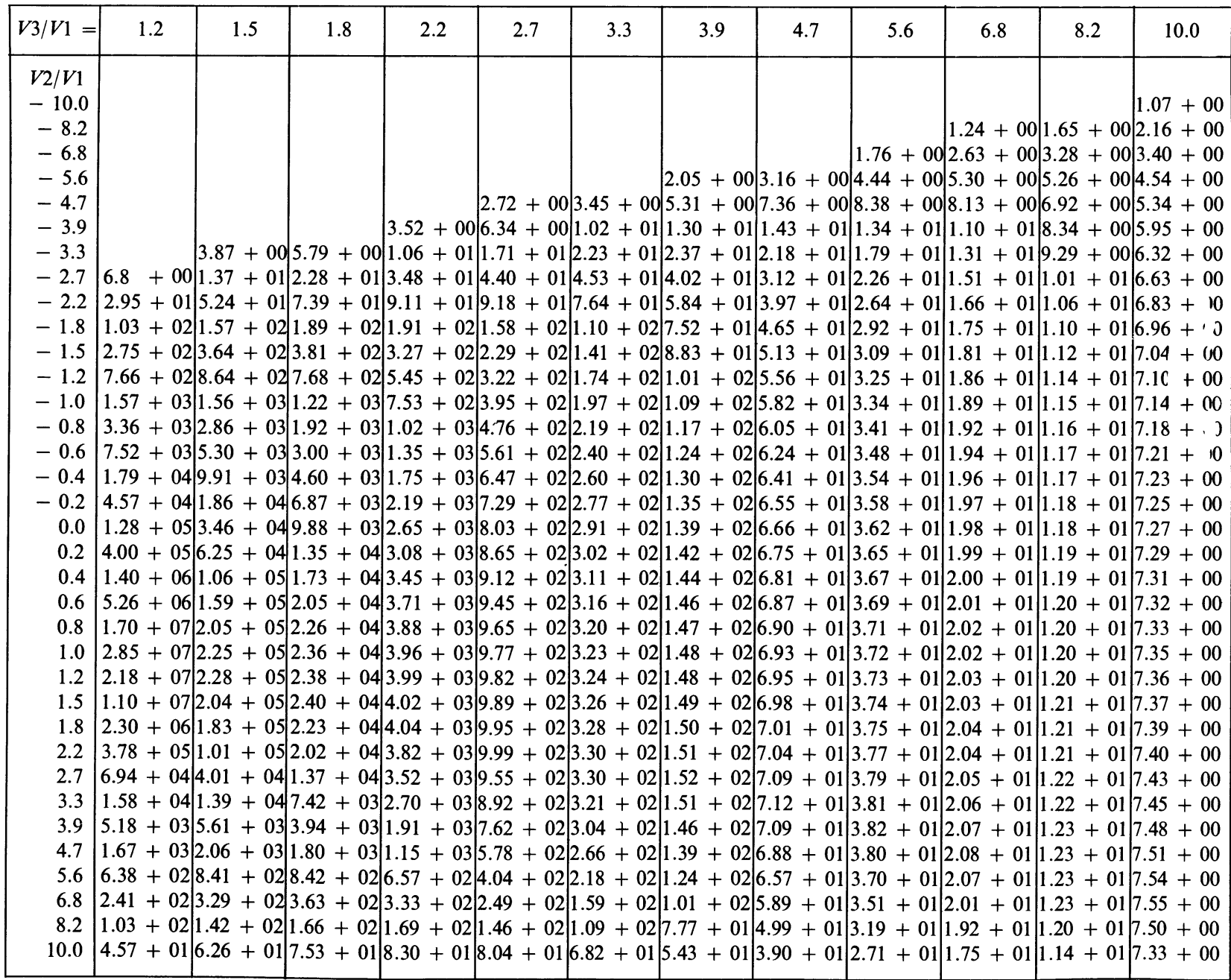


unipotential lenses, that his values are systematically slightly less than ours by a few tenths of a percent. So, we do not give tables of optical constants for these two lenses since they are practically the same as in Read's book.

The mechanical asymmetry introduces a new parameter in the values of the optical constants. We have not computed systematically every possible lens, but a large number of lenses with $V 1, V 2, V 3, g, d$ and $x$ chosen at random and we show a few typical results in figure 5 . We have plotted the $F 1$ and $S P 1$ values obtained by altering the shape of the lens $g=d=0.2$, leaving $x$ and either $g$ or $d$ constant. The same conclusion can be drawn from this figure as from figures 3 and 4 . In a first approximation, the two quantities $F 1$ and $S P 1$ do not depend on the mechanical asymmetry of the lens, provided that $x$ remains constant. In a second approximation, the correction depends on the position of the asymmetry (right or left), but it is slight and proportional to $g-x$ or $d-x$.

7. Conclusion. - In this paper, we have presented a simple accurate method of computing the potential within three-cylinder electrostatic lenses. We have achieved an analytical expression of it which can be computed at any point within the lens, and whose derivatives can be obtained easily and accurately.

We have computed the optical constants of mechanically symmetrical lenses for various values of the cylinder potentials. The results agree very well with those obtained by Read by means of the charge distribution.

We have also studied the influence of the mechanical asymmetry on the optical constants and we have shown an important property of these lenses : the optical properties mainly depend on the distance between the median planes of the gaps and only slightly on the lengths of these gaps.

Acknowledgments. - All the numerical calculations have been carried out on the UNIVAC 1110 computer in the Calculation Centre of Paris-Sud University, and I thank its technical staff for their help in programming.

ERRATA. - In table I, Part 1, for $z<0, V_{2}(r, z)$ has to be multiplied by 2 , and $V_{3}(r, z)$ by 6 .

Table VII. - The spherical aberration constant $S P 2$ of the same lens as a function of the ratios $V 2 / V 1$ and $V 3 / V 1$.

\begin{tabular}{|c|c|c|c|c|c|c|c|c|c|c|c|c|}
\hline$V 3 / V 1=$ & 1.2 & 1.5 & 1.8 & 2.2 & 2.7 & 3.3 & 3.9 & 4.7 & 5.6 & 6.8 & 8.2 & 10.0 \\
\hline$V 2 / V 1$ & & & & & & & & & & & & \\
\hline-10.0 & & & & & & & & & & & & $2.95-01$ \\
\hline-8.2 & & & & & & & & & & $5.26-01$ & $4.76-01$ & $4.14-01$ \\
\hline-6.8 & & & & & & & & & $7.58-01$ & $8.06-01$ & $7.19-01$ & $5.17-01$ \\
\hline-5.6 & & & & & & & $1.15+00$ & $1.36+00$ & $1.48+00$ & & $.49-$ & $5.83-01$ \\
\hline-4.7 & & & & & $1.92+00$ & $1.95+00$ & $2.45+00$ & $2.67+00$ & $2.38+00$ & $1.73+$ & $1.10+$ & $6.13-01$ \\
\hline-3.9 & & & & $2.55+00 \mid$ & $3.80+00$ & $4.98+00$ & $5.23+00 \mid$ & $4.55+00$ & $3.37+00 \mid$ & $2.08+$ & $1.19+0$ & $6.26-01$ \\
\hline-3.3 & & $3.36+00$ & $4.46+00$ & $6.97+00$ & $9.32+00$ & $9.90+00$ & $8.72+00$ & $6.36+00$ & $4.13+00$ & $2.30+$ & $1.24+0$ & $6.30-01$ \\
\hline-2.7 & $6.30+00$ & $1.12+01$ & $1.63+01$ & $2.11+01$ & $2.22+01$ & $1.85+01$ & $1.36+01$ & $8.36+00 \mid$ & $4.82+00$ & $2.47+00$ & $1.27+00$ & $6.30-01$ \\
\hline-2.2 & $.67+01$ & $4.09+01$ & $5.03+01$ & $5.23+01$ & $4.35+$ & $2.93+01$ & $1.85+$ & $1.00+01$ & $5.30+00$ & $2.56+$ & $1.28+00$ & $6.28-01$ \\
\hline-1.8 & $.22+01$ & $1.19+02$ & $1.24+02$ & $1.06+02$ & $7.14+01$ & $4.04+01$ & $2.27+01$ & $1.12+01$ & $5.59+00$ & $2.61+00$ & $1.28+00$ & $6.27-01$ \\
\hline-1.5 & $.43+02$ & $2.71+02$ & $2.45+02$ & $1.75+02$ & $1.00+02$ & $4.97+01$ & $2.57+01$ & $1.19+01$ & $5.76+00$ & $2.63+00$ & $1.28+0$ & $6.26-01$ \\
\hline-1.2 & $.70+02$ & $6.33+02$ & $4.82+02$ & $2.84+02$ & $1.37+02$ & $5.94+01$ & $2.85+01$ & $1.25+01$ & $5.88+00$ & $2.65+00$ & $1.28+00$ & $6.26-01$ \\
\hline-1.0 & $37+03$ & $1.13+03$ & $7.55+02$ & $3.86+02$ & $1.65+$ & $58+01$ & $3.02+01 \mid$ & $1.28+01$ & $5.94+00$ & $2.65+$ & $1.28+00 \mid$ & $6.26-01$ \\
\hline-0.8 & $.90+03$ & $2.05+03$ & $1.17+03$ & $5.14+02$ & $1.94+02$ & $7.18+01$ & $3.16+01$ & $1.31+01$ & $5.99+00$ & $2.66+00$ & $1.28+00$ & $6.26-01$ \\
\hline-0.6 & $.47+03$ & $3.76+03$ & $81+03$ & $6.71+02$ & 2 & $72+01$ & $3.28+01$ & $1.33+01$ & $6.02+00$ & $2.66+00$ & $1.28+0$ & $6.26-01$ \\
\hline-0.4 & $.53+04$ & $6.97+03$ & $2.73+03$ & $8.52+02$ & $2.55+02$ & $8.20+01$ & $3.38+01$ & $1.35+01$ & $6.05+00 \mid$ & $2.66+00$ & $1.28+00$ & $6.26-01$ \\
\hline-0.2 & $.90+04$ & $1.30+04$ & $4.03+03$ & $1.05+03$ & $2.82+02$ & $59+01$ & $3.46+01$ & $1.36+01$ & $6.07+00$ & $2.66+00$ & $1.28+00$ & $6.27-01$ \\
\hline 0.0 & $.09+05$ & $2.39+04$ & $5.72+03$ & $1.25+03$ & $3.06+02$ & $8.90+01$ & $3.52+01$ & $1.37+01$ & $6.08+00$ & $2.67+00$ & $1.28+00$ & $6.28-01$ \\
\hline 0.2 & $.38+05$ & $4.28+04$ & $7.72+03$ & $1.44+03$ & $3.24+02$ & $\mid 9.13+01$ & $3.56+01 \mid$ & $1.37+01$ & $6.09+00$ & $2.67+00$ & $1.29+00$ & $6.29-01$ \\
\hline 0.4 & $18+06$ & $6+04$ & $9.77+03$ & $1.59+03$ & $3.38+02$ & $9.28+01$ & $3.59+01$ & $1.38+01$ & $6.10+00$ & $2.67+00$ & $1.29+0$ & $6.31-01$ \\
\hline 0.6 & $.41+06$ & $1.07+05$ & $1.15+04$ & $1.69+03$ & $3.47+02$ & $9.38+01$ & $3.60+01$ & $1.38+01$ & $6.11+00$ & $2.68+00$ & $1.29+00$ & $6.32-01$ \\
\hline 0.8 & $42+07$ & $7+05$ & $6+04$ & & & $4+01$ & $2+01$ & $1.38+01$ & & 2 & $29+0$ & $34-01$ \\
\hline 1.0 & $38+0$ & $1.50+05$ & $1.30+04$ & $1.78+03$ & $3.55+02$ & $9.49+01$ & $3.63+01$ & $1.39+01$ & $6.14+00$ & 2.6 & $1.30+00$ & $6.37-01$ \\
\hline 1.2 & $80+0$ & $1.52+0$ & $1.32+04 \mid$ & $1.79+03$ & & $3+01$ & $64+01$ & $1.39+01$ & $6+00$ & 2. & $1.30+0$ & $.39-01$ \\
\hline 1.5 & $15+06$ & $1.35+05$ & $|1.33+04|$ & $1.81+03$ & $3.60+02$ & $9.60+01$ & $3.67+01 \mid$ & $1.40+01$ & $6.20+00$ & $2.72+00$ & $1.31+00$ & $6.42-01$ \\
\hline 1.8 & $90+06$ & $1.21+05$ & $1.23+04$ & $1.83+03$ & $3.64+02$ & $9.69+01$ & $70+01$ & $1.41+01$ & $25+00$ & 2.74 & $2+0$ & $6.46-01$ \\
\hline 2.2 & $10+05$ & $6.62+04$ & $1.11+04$ & $1.71+03$ & $3.66+02$ & $\mid 9.80+01$ & $3.74+01$ & $1.43+01$ & $6.31+00 \mid$ & $2.76+00$ & $1.33+00$ & $6.52-01$ \\
\hline 2.7 & $67+04$ & $2.59+04$ & $7.36+03 \mid$ & $1.56+03$ & $3.46+02$ & $9.81+01$ & $3.78+01 \mid$ & $1.45+01$ & $6.39+00$ & $2.80+$ & $1.35+00$ & $6.59-01$ \\
\hline 3.3 & $.28+04$ & $8.87+03$ & $3.92+03$ & $1.17+03$ & $3.19+02$ & $\mid 9.44+01$ & $3.75+01 \mid$ & $1.46+01$ & $6.46+00$ & $2.83+00 \mid$ & $1.36+00$ & $6.67-01$ \\
\hline 3.9 & $19+03$ & $3.53+03$ & $2.05+03$ & $8.12+02 \mid$ & $2.67+02$ & $8.79+01$ & $3.58+01 \mid$ & $1.44+01$ & $6.47+00$ & $2.85+00$ & $1.38+00$ & $6.74-01$ \\
\hline 4.7 & $35+03$ & $1.28+03$ & $9.19+02$ & $4.76+02$ & $1.96+02$ & $7.49+01$ & $3.33+01 \mid$ & $1.38+01$ & $6.39+00$ & $2.85+00$ & $1.39+00$ & $6.81-01$ \\
\hline 5.6 & $10+02$ & $5.16+02$ & $4.21+02$ & $2.65+02$ & $1.33+02$ & $5.93+01$ & $2.89+01$ & $1.29+01$ & $6.10+00$ & $2.81+00$ & $1.38+00$ & $6.85-01$ \\
\hline 6.8 & $.92+02$ & $2.00+02$ & $1.78+02 \mid$ & $1.31+02$ & $7.93+01$ & $4.17+01$ & $2.27+01 \mid$ & $1.11+01$ & $5.63+00 \mid$ & $2.68+00$ & $1.36+00$ & $6.82-01$ \\
\hline 8.2 & $19+01$ & $8.55+01$ & $8.01+01$ & $6.51+01$ & & $2.74+01 \mid$ & $1.67+01$ & $9.01+00$ & $4.91+00$ & $2.48+00$ & $1.29+00$ & $6.68-01$ \\
\hline 10.0 & $3.62+01$ & $3.75+01$ & $3.60+01 \mid$ & $3.12+01 \mid$ & $2.40+01$ & $|1.65+01|$ & $|1.11+01|$ & $6.71+00$ & $|3.97+00|$ & $2.16+00 \mid$ & $1.19+00$ & $6.34-01$ \\
\hline
\end{tabular}




\section{References}

[1] Saito, T., Kubota, S. and Miyaoka, S., J. Appl. Phys. 44 (1973) 4505-4510.

[2] Saito, T. and OJars, J. Sovers, J. Appl. Phys. 48 (1977) 23062311.

[3] Adams, A. and Read, F. H., J. Phys. 55 (1972) 150-155.

[4] Adams, A. and Read, F. H., J. Phys. 5 (1972) 156-160.

[5] Van Der Merwe, J. P., J. Appl. Phys., 49 (1978) 4335-4340.

[6] Bonjour, P., Revue Phys. Appl. 14 (1979) 533-540.
[7] Bertram, S., J. Appl. Phys. 13 (1942) 496-502.

[8] Grivet, P., Electron Optics (Pergamon, Oxford) 1965

[9] Slodzian, G. and Figueras, A., J. Physique Lett. 39 (1978) L 90-L 93.

[10] ReAd, F. H. and Harting, E., Electrostatic Lenses (Elsevier, Amsterdam) 1976

[11] Heddle, D. W. O. and Kurepa, M. V., J. Phys. 23 (1970) 552-554. 